\title{
Combination of dasatinib and quercetin improves cognitive abilities in aged male Wistar rats, alleviates inflammation and changes hippocampal synaptic plasticity and histone $\mathrm{H3}$ methylation profile
}

\author{
Adam Krzystyniak ${ }^{1, *}$, Malgorzata Wesierska ${ }^{2,}{ }^{,}$, Gregory Petrazzo ${ }^{1}$, Agnieszka Gadecka $^{1}$, \\ Magdalena Dudkowska' ${ }^{1}$, Anna Bielak-Zmijewska ${ }^{1}$, Grazyna Mosieniak ${ }^{1}$, Izabela Figiel ${ }^{3}$, Jakub \\ Wlodarczyk ${ }^{3}$, Ewa Sikora ${ }^{1}$ \\ ${ }^{1}$ Laboratory of Molecular Bases of Aging, Nencki Institute of Experimental Biology, Polish Academy of Sciences, \\ Warsaw 02-093, Poland \\ 2Laboratory of Neuropsychology, Nencki Institute of Experimental Biology, Polish Academy of Sciences, Warsaw 02-093, \\ Poland \\ ${ }^{3}$ Laboratory of Cell Biophysics, Nencki Institute of Experimental Biology, Polish Academy of Sciences, Warsaw 02-093, \\ Poland \\ ${ }^{*}$ Equal contribution
}

Correspondence to: Ewa Sikora, Jakub Wlodarczyk; email: e.sikora@nencki.edu.pl, j.wlodarczyk@nencki.edu.pl Keywords: aging, brain, plasticity, cognition, memory, hippocampus, SASP, senescence

Received: October 18, $2021 \quad$ Accepted: January 11, 2022

Published: January 18, 2022

Copyright: (c) 2022 Krzystyniak et al. This is an open access article distributed under the terms of the Creative Commons Attribution License (CC BY 3.0), which permits unrestricted use, distribution, and reproduction in any medium, provided the original author and source are credited.

\section{ABSTRACT}

Aging is associated with cognitive decline and accumulation of senescent cells in various tissues and organs. Senolytic agents such as dasatinib and quercetin $(D+Q)$ in combination have been shown to target senescent cells and ameliorate symptoms of aging-related disorders in mouse models. However, the mechanisms by which senolytics improve cognitive impairments have not been fully elucidated particularly in species other than mice. To study the effect of senolytics on aging-related multifactorial cognitive dysfunctions we tested the spatial memory of male Wistar rats in an active allothetic place avoidance task. Here we report that 8 weeks treatment with $D+Q$ alleviated learning deficits and memory impairment observed in aged animals. Furthermore, treatment with $D+Q$ resulted in a reduction of the peripheral inflammation measured by the levels of serum inflammatory mediators (including members of senescent cell secretome) in aged rats. Significant improvements in cognitive abilities observed in aged rats upon treatment with $D+Q$ were associated with changes in the dendritic spine morphology of the apical dendritic tree from the hippocampal CA1 neurons and changes in the level of histone H3 trimethylation at lysine 9 and 27 in the hippocampus. The beneficial effects of $D+Q$ on learning and memory in aged rats were long-lasting and persisted at least 5 weeks after the cessation of the drugs administration. Our results expand and provide new insights to the existing knowledge associated with effects of senolytics on alleviating age-related associated cognitive dysfunctions.

\section{INTRODUCTION}

Aging is the major risk factor for cancer, cardiovascular disease, diabetes, and neurodegenerative disorders associated with multiple cognitive impairments that lead to significant disabilities and lower quality of life.
According to the UN estimates, the world population of people above 65 years old will double in the next 30 years and is expected to exceed 1,5 billion worldwide by 2050 (UN, 2019). However, if the prevalence of the COVID-19 infection continues to grow, this could impact life expectancy and change these estimations [1]. 
Aging is associated with cognitive changes that have been well described in both humans and laboratory animals. There are different aspects of cognition that gradually decline with age including, short- and longterm memory, learning, conceptual reasoning, processing speed, and psychomotor abilities [2]. Since normal aging has not been associated with significant changes in the number of brain cells [3], the accompanying cognitive impairments are believed to result from alterations in the cellular and molecular mechanisms of brain plasticity. Dendritic protrusions called dendritic spines that form synapses have distinct functional characteristics depending on their shape. The longer and thinner dendritic spines are immature and represent new, more unstable connections, whereas bigger and mushroom-like spines usually are associated with stable synapses. Both, the least mature and most mature spines are underrepresented in the aged hippocampus and frontal cortex, two brain regions critical for cognitive abilities [4, 5]. The exceptional plasticity of mature neurons is controlled by activitydependent changes in gene expression. One of the mechanisms that allow rapid changing of the accessibility of genes to the transcriptional machinery is post-translational modification of histones such as methylation. Methylation of histones, primarily of their lysine $(\mathrm{K})$ residues, regulates memory formation and synaptic plasticity [6]. In particular, methylation of histone $\mathrm{H} 3$ has been implicated in age-associated cognitive decline in mice. Aging causes upregulation of $\mathrm{H} 3$ trimethylation at lysine 3 (H3K9me3) [7] and downregulation at lysine 27 (H3K27me3) [8] in the mouse hippocampus. Recently it has been shown that the decrease in the $\mathrm{H} 3 \mathrm{~K} 9 \mathrm{me} 3$ by systemic administration of an inhibitor of the principal enzyme responsible for the trimethylation of $\mathrm{H} 3 \mathrm{~K} 9$ (histone methyltransferase SUV39H1) improved memory performance in aged, but not young mice [9].

It has been postulated that the accumulation of senescent cells with age may be the main culprit of aging. Indeed, the accumulation of senescent cells was observed in healthy tissue of old animals and humans and at sites of many age-related pathologies, including the brains of animal models of Parkinson's and Alzheimer's diseases (reviewed in [10]). Proinflammatory cytokines such as TNF- $\alpha$, IL-6 and IL1 , which are the components of so termed senescenceassociated secretory phenotype - SASP when constitutively secreted by senescent cells [11], participate in a low grade inflammatory state, termed inflammaging [12]. Inflammaging may be one of the major mechanisms responsible for diseases of advanced age. Multiple studies have shown that elevated levels of inflammatory cytokines regulate learning and memory by modulating synaptic plasticity (e.g. $[13,14])$.
Senolytics are synthetic or natural compounds, which are able to target senescent cells $[15,16]$. Their primary mechanism of action relies on inducing apoptotic cell death by affecting several different pro-survival pathways [17]. However, it is postulated that those compounds may also exert senostatic activity, by inhibiting SASP, which otherwise leads to spreading of senescence due to the paracrine effect of senescence progression [18]. Till now several published animal studies have shown that the eradication of senescent cells alleviates many aging-related pathologies [19-21]. The natural senolytic fisetin and a combination of dasatinib and quercetin $(\mathrm{D}+\mathrm{Q})$ treatment improve cognitive ability in mouse models of Alzheimer's disease and dementia $[22,23]$. Fisetin or $\mathrm{D}+\mathrm{Q}$ appear to be the most effective senolytics tested in many preclinical studies and growing body of clinical trials $[20,24]$. In a recent study Ogrodnik et al. provided the first evidence for beneficial effects of $D+Q$ in alleviating age-associated cognitive decline in mice, tested in water - escape version of the Stone T-maze [25]. In the present study, we hypothesized that $\mathrm{D}+\mathrm{Q}$ treatment alleviates cognitive decline in rats by decreasing senescence-associated phenotype burden. To verify this hypothesis, we have used a method that allows complex analysis of different aspects of cognition, namely spatial learning and memory, in the active allothetic place avoidance task (AAPAT). In the AAPAT test, formation of spatial representation requires a fully functional hippocampus [26]. To better understand the mechanism of action of $\mathrm{D}+\mathrm{Q}$ we sought to investigate whether improved cognition following $\mathrm{D}+\mathrm{Q}$ treatment in aged animals is associated with changes in the peripheral level of inflammation, brain synaptic plasticity and regulation of gene expression involved in modulation of synaptic plasticity. Our results provide new insights into the mechanisms of improvement of cognitive abilities by senolytics and offer valuable data confirming the effectiveness of $D+Q$ treatment for age-associated learning and memory deficits in rats.

\section{RESULTS}

\section{$D+Q$ alleviates age-associated cognitive deficits in aged rats}

To study the effect of $\mathrm{D}+\mathrm{Q}$ on aging-associated cognitive impairment, we designed an experiment in which allothetic spatial memory was tested in young and aged male Wistar rats (GROUP 1) in the active allothetic place avoidance task (AAPAT). The AAPAT was used to assess the acquisition of allothetic spatial memory before (1st TRAINING) and after the treatment with $\mathrm{D}+\mathrm{Q}$ or vehicle (2nd TRAINING). The experimental outline is depicted in Figure 1A. It 
consisted of two place avoidance trainings, 5 training days each spaced by 8 weeks of vehicle or $D+Q$ treatment period. It is important to notice that the position of the shock place during the 2nd TRAINING was described by new room frame coordinates in comparison to the 1st TRAINING. It therefore required the formation of a new spatial memory.

\section{Aged rats presented worse memory acquisition in the place avoidance than young rats during the 1st TRAINING}

We observed that aged non-treated rats assigned to vehicle or $\mathrm{D}+\mathrm{Q}$ treatment groups (during $1 \mathrm{st}$ TRAINING - before treatment), learned the place avoidance in the AAPAT worse than young non-treated rats assigned to the respective vehicle or $\mathrm{D}+\mathrm{Q}$ treatment groups and exhibited short-term memory impairment. The aged animals assigned to vehicle or $\mathrm{D}+\mathrm{Q}$ treatment groups (during 1st TRAINING - before treatment), entered more often into the to-be-avoided place (made errors) and received more shocks than young rats assigned to the respective vehicle or $\mathrm{D}+\mathrm{Q}$ treatment groups. The two-way ANOVA (4 groups and 5 days) for number of entrances and shocks confirmed main effect of groups (entrances $\mathrm{F}_{(3,26)}=9.718 ; p=0.002$; shocks $\mathrm{F}_{(3,25)}=21.60 ; p<0.001$ ) (Figure 1B I, 1C I). The value of each parameter depended on the day of the training and significantly changed during the training, which was confirmed by the two-way ANOVA for days. A detailed description of results for days is presented in the Supplementary Data (Supplementary Data 1).

Performance of place avoidance engages executive functions described by skill learning which was measured by shocks to entrances ratio (SHs/ENTRs). Low value of the ratio indicates effective skill learning. We found that aged rats presented worse skill learning than young rats. The two-way ANOVA (4 groups and 5 days) for SHs/ENTRs ratio confirmed main groups $\left(\mathrm{F}_{(3,26)}=34.61 ; p<0.001\right)$ and days effect $\left(\mathrm{F}_{(4,104)}=\right.$ $6.65 ; p<0.001$ ) (Figure 1D I).

Aged rats presented worse short-term memory than young rats. It was manifested as shorter maximum time avoided between two entries into the shock place in aged rats. The same ANOVA for maximum time avoided confirmed the main effect of groups $\left(\mathrm{F}_{(3,26)}=\right.$ $4.19 ; p=0.015)$ and days of training $\left(\mathrm{F}_{(4,104)}=5.57\right.$; $p<0.001$ ) (Figure 1E).

Similarly, time to the first entrance (T1) differed between young and aged rats assigned to their respective vehicle or $\mathrm{D}+\mathrm{Q}$ treatment groups. The twoway ANOVA (4 groups and 5 days) confirmed main groups $\left(\mathrm{F}_{(3,22)}=3.471 ; p=0.033\right)$ and days effect $\left(\mathrm{F}_{(4,88)}=3.59 ; p=0.009\right)$ (Figure 1E). Tukey's post hoc multiple comparisons test for groups confirmed that both vehicle- and $\mathrm{D}+\mathrm{Q}$ - treated young rats presented a longer $\mathrm{T} 1$ than aged rats assigned to vehicle and $\mathrm{D}+\mathrm{Q}$ treatment groups $(p=0.056)$.

No differences were found between young rats assigned to the vehicle treatment group and young rats assigned to the $\mathrm{D}+\mathrm{Q}$ treatment groups (the same applies to aged rats). On D1 length of time to the first entry was shorter than on D4 (Tukey's multiple comparisons test; $p=$ 0.02) (Figure 1).

Aged rats improved place avoidance acquisition and short-term memory after administration of $D+Q$

After 8 weeks of $\mathrm{D}+\mathrm{Q}$ or vehicle administration the AAPAT was repeated with the new location of the shock place. Change of the shock place demanded formation of a new spatial representation of a to-beavoided place (2nd TRAINING) (Figure 1A). D+Q treatment improved cognitive ability only in aged rats. Aged rats in the $\mathrm{D}+\mathrm{Q}$ group exhibited significant place avoidance performance and short-term memory improvement relative not only to the vehicle group but also to their own results from pretreatment training (1st TRAINING).

The two-way ANOVA ( 2 groups and 5 days; aged vehicle 2nd TRAINING, aged D+Q 2nd TRAINING vs. 5 days) confirmed main effect of group for shocks $\left(\mathrm{F}_{(1,11)}=7.02 ; p=0.022\right)$ in the 2nd TRAINING for aged vehicle and aged $\mathrm{D}+\mathrm{Q}$ groups of rats. Aged $\mathrm{D}+\mathrm{Q}$ rats received less shocks than aged VEH treatment rats (Figure 1C II). Moreover, aged $\mathrm{D}+\mathrm{Q}$ treated rats presented effective skill learning (SHs/ENTRs) in comparison to aged VEH treated rats $\left(\mathrm{F}_{(1,11)}=9.1 ; p=\right.$ 0.012) independent on training days (days effect NS) (Figure 1B II-1D II). Aged rats from the vehicle and $\mathrm{D}+\mathrm{Q}$ group presented similar maximum time avoided and time to the first entrance on 2nd training (Figure 1E II and 1F II) (Supplementary Data 2).

Performance of aged rats in AAPAT before $\mathrm{D}+\mathrm{Q}$ treatment was significantly different from that after $\mathrm{D}+\mathrm{Q}$ treatment. The two-way ANOVA ( 2 trainings 1 st TRAINING, 2nd TRAINING and 5 days) for entrances, shocks, skill learning and maximum time avoided confirmed main effect of trainings (entrances $\mathrm{F}_{(1,11)}=$ 9.87; $p=0.009$; shocks $\mathrm{F}_{(1,11)}=12.66 ; p=0.005$; SHs/ENTRs ratio $\left.\mathrm{F}_{(1,11)}=8,87 ; p=0.013\right)$ and maximum time avoided $\left.\mathrm{F}_{(1,11)}=6.15 ; p=0.03\right)$. Aged rats during 2 nd TRAINING after $\mathrm{D}+\mathrm{Q}$ treatment made a smaller number of ENTR, received less shocks and presented a low value of SHs/ENTRs ratio and a longer maximum time avoided compared to 1st TRAINING (before $\mathrm{D}+\mathrm{Q}$ treatment) (Figure 1B I-1E I). Moreover, old rats from the $\mathrm{D}+\mathrm{Q}$ group during 2 nd TRAINING 


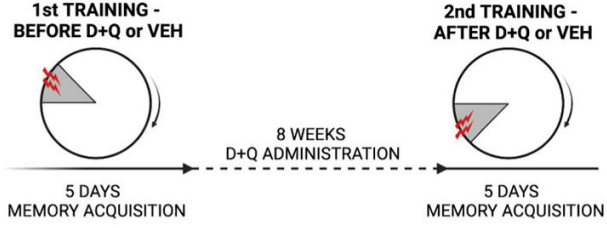

$1^{\text {st }}$ TRAINING vs $2^{\text {nd }}$ TRAINING

$2^{\text {nd }}$ TRAINING
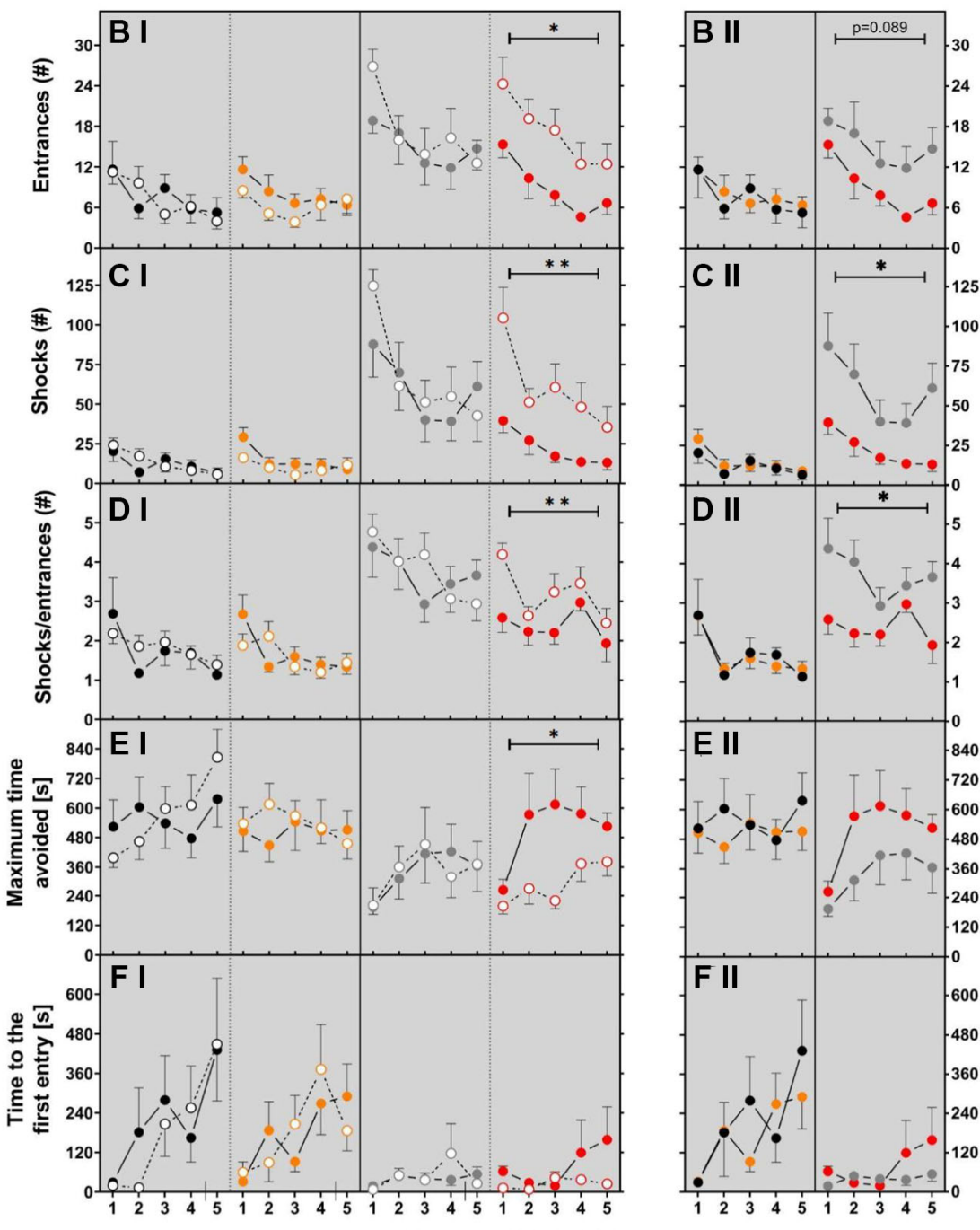

Days of TRAINING
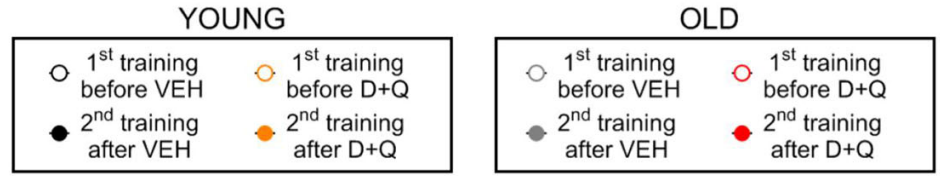

Figure 1. Treatment with $D+Q$ improved cognitive ability in aged rats tested in the AAPAT on spatial memory. (A) Schematic diagram of the experimental design. Experiments consisted of 2 trainings, each with a different position of to-be-avoided place. The number of entries into the shock place (\# of entries, B), the number of shocks (\# shocks, C) and the number of shocks per one entry ratio (Shocks/entrance, $\mathbf{D}$ ), together with maximum time avoided (E) and time to the first entry (F) during place avoidance training reflect changes in the learning abilities and memory in aged rats. Performance of place avoidance by young (3-mo old; YOUNG; $N=7$ ) (black - vehicle, orange - $\mathrm{D}+\mathrm{Q}$ ) and aged (22-mo old; OLD; $N=7$ ) (grey - vehicle, red - D+Q) rats in the AAPAT before and after 8 weeks of treatment with $D+Q$ or vehicle $(V E H)$ by oral gavage. Figures labeled with "I" depict comparison between 1st TRAINING and 2nd TRAINING, "II" between VEH and D+Q groups results of the 2 nd TRAINING. The results are expressed as mean \pm SEM, $N=7,{ }^{*} p<0.05$, ${ }^{* *} p<0.01,{ }^{* * *} p<0.001$. Data obtained for groups at consecutive trials were compared using repeated measures two-way ANOVA (2 groups and 5 days). 
compared to their own results from the 1st TRAINING showed significant day effects for all the above parameters (Supplementary Data 2).

$\mathrm{D}+\mathrm{Q}$ treatment did not influence performance of young rats neither when compared to the young vehicle group nor to their own results from the 1st TRAINING. The two-way ANOVA (2 groups and 5 days) for the number of entrances, shocks, shocks/entrances ratio and length of the maximum time avoided for young rats from the vehicle and $\mathrm{D}+\mathrm{Q}$ groups showed non-significant main effect of groups. Effect of days was significant (Supplementary Data 3). Young rats regardless of treatment, performed place avoidance on a similar level and improved it in consecutive days of training (Supplementary Data 4).

Moreover, long-term memory evaluated by the time to first entrance on the-to-be-avoided place in young rats from vehicle and D+Q group before treatment as well as between 1st TRAINING and 2nd TRAINING for young $\mathrm{D}+\mathrm{Q}$ rats was on a similar level (the same ANOVA shows non-significant groups, trainings or days effect) (Figure 1F I, 1F II).

$\mathrm{D}+\mathrm{Q}$ treatment had no significant effect on body mass or locomotor abilities in young rats regardless of treatment (Supplementary Figure 1).

\section{$D+Q$ treatment decreases peripheral inflammation including SASP factors in aged rats}

Senolytics has been shown to reduce peripheral inflammation which is elevated in aged animals [27]. In order to confirm that $\mathrm{D}+\mathrm{Q}$ treatment, in fact, alleviates low grade inflammation in rats we analyzed serum factors such as cytokine, chemokines and growth factors which belong to SASP [28]. As expected, aged rats showed generally higher inflammation, with analyzed serum factors being elevated compared to young rats (young vehicle vs. aged vehicle $\mathrm{F}_{(1,378)}=211.3 ; p<$ 0.001 and young $\mathrm{D}+\mathrm{Q}$ vs. aged $\mathrm{D}+\mathrm{Q} \mathrm{F}_{(1,351)}=51.90$; $p<0.001)$. Furthermore, $\mathrm{D}+\mathrm{Q}$ treatment resulted in significant decrease in the levels of peripheral inflammatory mediators in aged $\left(\mathrm{F}_{(1,338)}=8.038 ; p=\right.$ $0.005)$ but not young $\left(\mathrm{F}_{(1,364)}=0.4465 ; p=0.504\right)$ rats. However, $\mathrm{D}+\mathrm{Q}$ treatment did not result in a statistically significant change of any of the individual cytokines (Supplementary Figure 2). We did find significant differences in the levels of individual cytokines between young and aged rats, seven cytokines for vehicle treated groups (IL-1 $\alpha$, IL- $\beta$, IL-4, IL-2, IL-10, MCP-1 and TNF- $\alpha$ ) and only two for D+Q treated groups (IL-10 and TNF- $\alpha$ ) (Supplementary Figure 2). Interestingly IL10 , which is considered as the anti-inflammatory cytokine, was found to change on average in the opposite direction compared to other cytokines, further suggesting that $\mathrm{D}+\mathrm{Q}$ treatment promotes an antiinflammatory phenotype (Figure 2). In contrast to aged rats, serum level of cytokines and growth factors was not significantly affected by $\mathrm{D}+\mathrm{Q}$ treatment in young rats.

Improvement of cognitive skills in aged rats is associated with changes in synaptic plasticity in the stratum radiatum of CA1 region of the hippocampus

Synaptic plasticity is the activity-dependent modification of signal transmission between neurons. Changes in the synaptic transmission are associated with morphological alterations of the dendritic spines which constitute the postsynaptic part of the excitatory synapse. Synaptic plasticity, particularly in the hippocampal region, is critical for learning and memory formation. We hypothesized that improvement of cognitive abilities upon $\mathrm{D}+\mathrm{Q}$ treatment should therefore be associated with changes in synaptic plasticity. To test this hypothesis, we analyzed dendritic spines morphology and density in DiI stained sections of hippocampus from aged vehicle and $\mathrm{D}+\mathrm{Q}$ treated rats (Figure 3A and 3B). We found that dendritic spines on apical dendrites of the CA1 neurons underwent morphological changes after treatment with $\mathrm{D}+\mathrm{Q}$. The spines were longer $(p=0.008)$, and the ratio between the spine length and head width was significantly higher $(p=0.002)$ compared to the vehicle treated group. Moreover, we found a trend toward increase of the overall size of spines in the $\mathrm{D}+\mathrm{Q}$ group as measured by the circumference and area of spines (circumference $p=$ 0.055; area $p=0.079$ ) (Figure 3C). Interestingly, these changes were specific to apical dendrites as we did not observe similar changes in basal dendrites of CA1 neurons between $\mathrm{D}+\mathrm{Q}$ and vehicle groups of aged rats (length $p=0.209$; length to width $p=0.453$; circumference $p=0.364$; area $p=0.186$ ) (Figure 3D). Dendritic spines density was not affected by $\mathrm{D}+\mathrm{Q}$ treatment neither in the stratum oriens $(p=0.692)$ nor in the stratum radiatum $(p=0.443)$ (Figure $3 \mathrm{E}$ and $3 \mathrm{~F})$.

\section{Beneficial effects of $D+Q$ on cognitive skills in aged rats are accompanied by changes in histone $\mathrm{H3}$ methylation in the hippocampus}

Altered epigenetic profile has been associated with agedependent disorders. Methylation of histones H3 typically occurring at specific lysine $(\mathrm{K})$ residues, such as $\mathrm{H} 3 \mathrm{~K} 9, \mathrm{H} 3 \mathrm{~K} 27$ regulates chromatin state and plays an important role in the regulation of gene expression. In particular, trimethylation of $\mathrm{H} 3 \mathrm{~K} 9$ (H3K9me3) and H3K27 (H3K27me3) which regulates silent heterochromatin stability has been implicated in agedependent cognitive impairment [7, 8]. We 
hypothesized that rejuvenating effects of $\mathrm{D}+\mathrm{Q}$ would affect the histone $\mathrm{H} 3$ methylation profile. To test this hypothesis, we performed Western blot analysis of histone extracts from hippocampal lysates of aged D+Q or vehicle treated rats. Our results showed a significant decrease in $\mathrm{H} 3 \mathrm{~K} 9 \mathrm{me} 3$ levels relative to the total $\mathrm{H} 3$ in aged animals treated with $\mathrm{D}+\mathrm{Q}(p<0.001)$ (Figure 4A). Conversely, the levels of $\mathrm{H} 3 \mathrm{~K} 27 \mathrm{me} 3$ were significantly higher in aged animals treated with $\mathrm{D}+\mathrm{Q}$ compared to vehicle treated rats $(p=0.009)$ (Figure 4B).

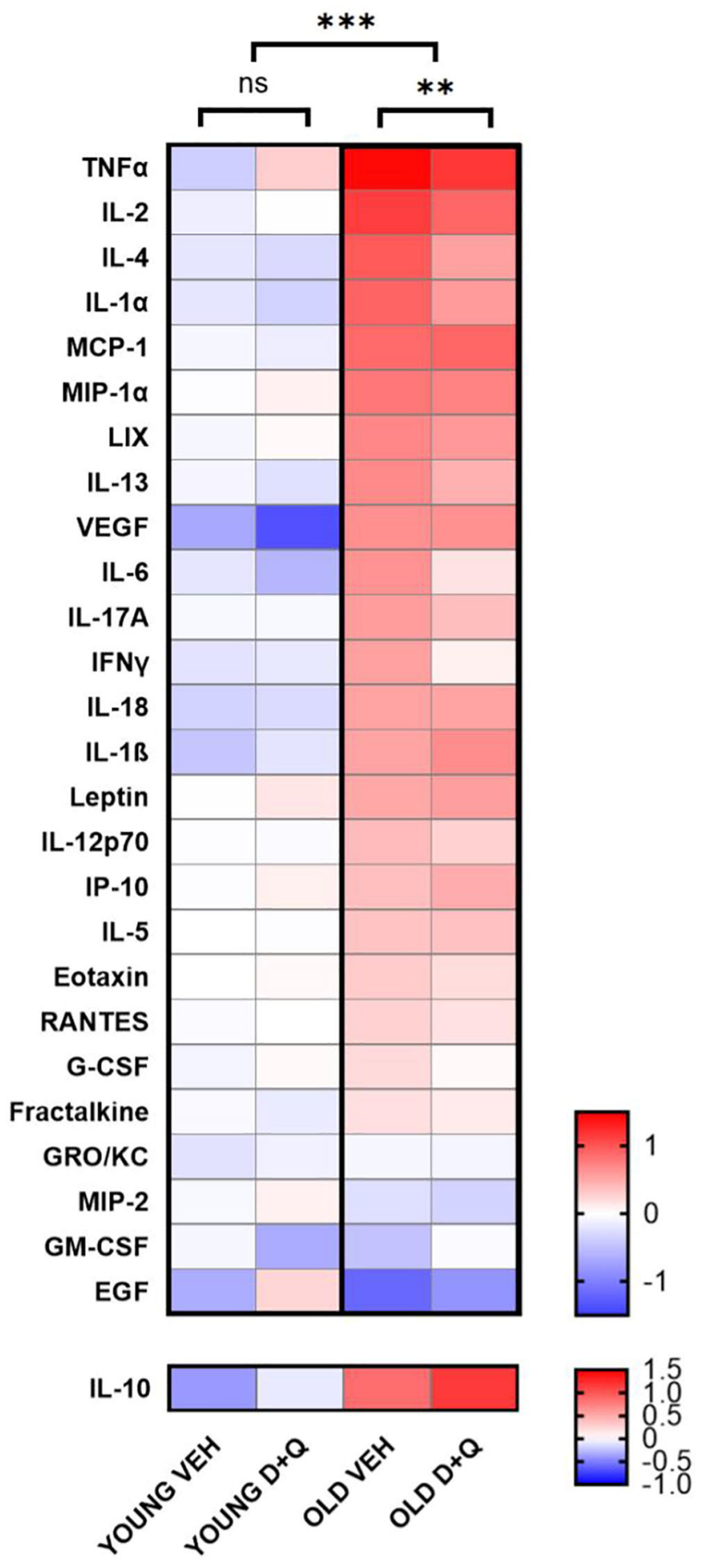

Figure 2. $\mathbf{D}+\mathbf{Q}$ treatment reduces peripheral inflammation in aged rats. The heat map depicts cytokines and growth factor levels in blood serum collected after the final behavioral test from young and aged (6-month-old and 25-month-old respectively at the time of collection) rats treated with $D+Q$ or vehicle (VEH). The results were normalized to the average of the young vehicle group. IL-10 as anti-inflammatory cytokine has been presented separately. The data were analyzed using two-way ANOVA; $n=7-8,{ }^{*} p<0.05$, ${ }^{* *} p<0.01,{ }^{* * *} p<0.001$. 
$D+Q$ alleviates age-associated cognitive deficits in aged rats at least 5 weeks after discontinuation of the treatment

To test whether the beneficial effect of $(D+Q)$ on cognitive functions is a long-lasting effect we performed another set of experiments in which aged and young Wistar rats (GROUP 2) were trained 3 times in the AAPAT, namely before $\mathrm{D}+\mathrm{Q}$ treatment (1st TRAINING), early after $\mathrm{D}+\mathrm{Q}$ administration (2nd TRAINING) and again 5 weeks ( 1 week of training +4 weeks being left undisturbed in their home cage) after discontinuation of $\mathrm{D}+\mathrm{Q}$ treatment (3rd TRAINING) (Figure 5A), each time to a new shock place.
A

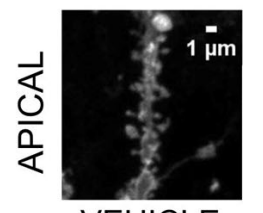

VEHICLE

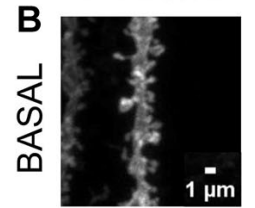

VEHICLE

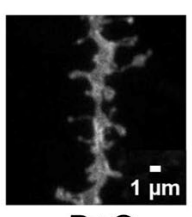

$D+Q$

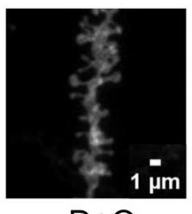

$D+Q$
C
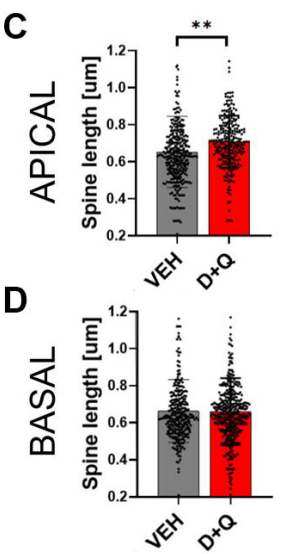

MORPHOLOGY
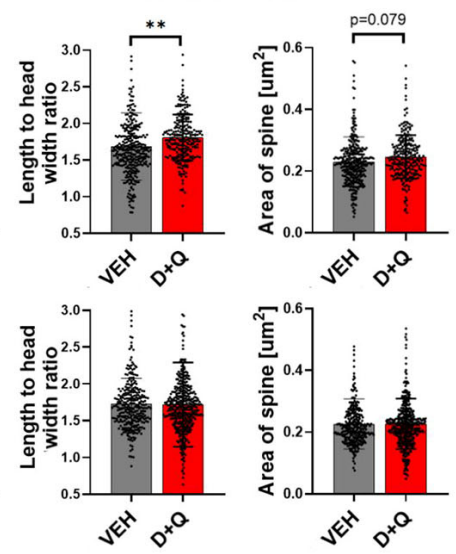

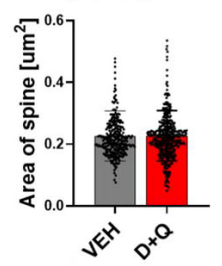

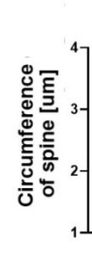
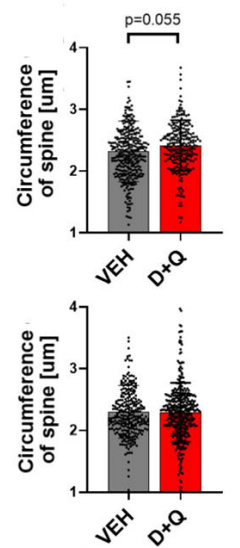

DENSITY

E

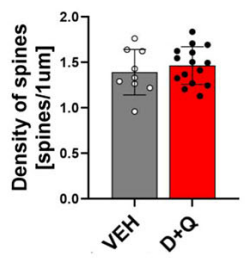

$\mathbf{F}$

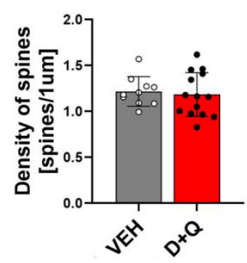

Figure 3. $D+Q$ treatment changes synaptic plasticity in the apical dendrites of neurons of the CA1 region of the hippocampus. Representative images of Dil stained dendrites (A and B). Dil stained hippocampal slices from aged vehicle (grey bars) or $D+Q$ (red bars) treated rats were used for the analysis of synaptic plasticity. Different parameters of the dendritic spine shape (length, length to width ratio, area, circumference $\mathbf{C}$ and $\mathbf{D})$ and spine density (E and $\mathbf{F})$ were analyzed in the CA1 region of the hippocampus. The analysis was done for two dendritic arbours of stratum pyramidale, namely basal (BASAL) ( $\mathbf{D}$ and $\mathbf{F}$ ) and apical (APICAL) dendrites (C and E). The data are expressed as mean \pm SEM, and analyzed using nested $t$-test. Dots on the bar plots represent values for single dendritic spines (C and D) or single image (E and F) $N_{\mathrm{VEH}}=3, N_{\mathrm{D}+\mathrm{Q}}=5$ animals, ${ }^{*} p<0.05,{ }^{* *} p<0.01,{ }^{* * *} p<0.001$.
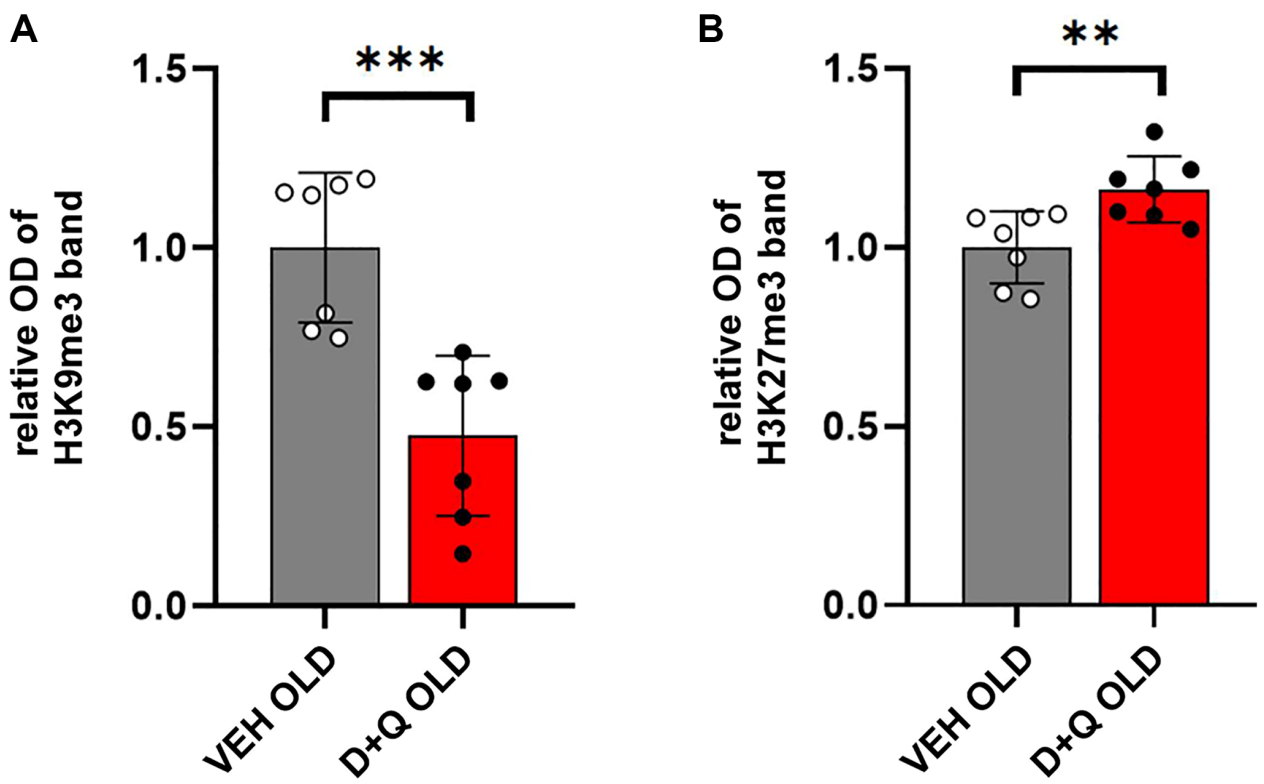

Figure 4. Changes in the levels of $\mathrm{H} 3$ histones in the hippocampus of aged rats after $\mathrm{D}+\mathrm{Q}$ treatment. Improved cognitive skills, decreased peripheral inflammation, and changes in neuronal synaptic plasticity are accompanied by changes in $\mathrm{H} 3$ methylation profile. Trimethylation of H3K9 (A) and H3K27 (B) relative to the whole H3 in the hippocampus of aged rats after D+Q or VEH treatment measured by Western blot. The data are expressed as means \pm SEM normalized to the average of the VEH group, and analyzed using $t$-test; $N=7,{ }^{*} p<$ $0.05,{ }^{* *} p<0.01,{ }^{* * *} p<0.001$. 
Similarly to the first set of experiments, we found that aged rats learned the place avoidance task worse than young rats before treatment with $\mathrm{D}+\mathrm{Q}$ during the 1 st TRAINING. In the 1st TRAINING aged rats made more entrances into the shock place, with shorter maximum time avoided and received more shocks compared to young rats. The two-way ANOVA (2 groups and 5 days) for entrances, shocks and maximum time avoided on the 1st TRAINING confirmed main effect of groups (entrances $\mathrm{F}_{(1,11)}=6.11 ; p<0.03$; shocks $\mathrm{F}_{(1,12)}=11.62 ; p=0.005 ;$ maximum time avoided $\left.\mathrm{F}_{(1,12)}=6.41 ; p=0.026\right)$. Performance of place avoidance changed across days (Supplementary Data 5).

The 2nd TRAINING of AAPAT with the new location of the to-be-avoided place started after 8 weeks of D+Q administration. During this training aged rats exhibited learning and memory improvements relative to their own results from the 1st TRAINING. (Figure 5B I5E I). The two-way ANOVA (2 trainings 1st TRAINING, 2nd TRAINING and 5 days) for the number of entrances, shocks, skill learning and maximum time avoided confirmed main effect of the training (entrances $\mathrm{F}_{(1,12)}=19.71 ; p<0.001$; shocks $\mathrm{F}_{(1}$, ${ }_{12)}=28.54 ; p<0,001$; SHs/ENTRs ratio $\mathrm{F}_{(1,12)}=19.00$; $p<0.001$; maximum time avoided $\mathrm{F}_{(1,12)}=16.12 ; p=$ $0.002)$. The performance of AAPAT improved across days as shown by the significant days effect described with details in (Supplementary Data 6).

The same ANOVA for young rats has shown a lack of significant differences between 1st TRAINING and 2nd TRAINING for entrance, shocks, skill learning and maximum time avoided. Similarly to the aged rats, the young rats improved performance in AAPAT across days of training (Supplementary Data 7).

Results of the 3rd TRAINING revealed that aged rats retained improvements in cognitive abilities at nearly the same level as immediately after $\mathrm{D}+\mathrm{Q}$ treatment (2nd TRAINING) (Figure 5B III-5E III). However, significant decrease in the number of entrances, number of shocks and increase of maximum time avoided were recorded during 3rd TRAINING in comparison to the 1st TRAINING in the group of aged rats (the same ANOVA (1st TRAINING, 3rd TRAINING $n=2$ vs. days $n=5$; entrances $\mathrm{F}_{(1,12)}=9.10 ; p=0,01$; shocks $\mathrm{F}_{(1,12)}=23.21 ; p<0.001 ;$ SHs/ENTRs ratio $\mathrm{F}_{(1,12)}=$ $15.13 ; p=0.002$; maximum time avoided $\mathrm{F}_{(1,12)}=9.96$; $p=0.008)$ (Figure 5B II-5E II). During 3rd TRAINING rats received less shocks than on D1 and D2 of 1st TRAINING (Supplementary Data 8).

Due to the long duration of the experiments, a group of young rats reached approximately $7-8$ months of age at the end of 3rd TRAINING when the final memory training to a new to-be-avoided place was performed. Treatment with $\mathrm{D}+\mathrm{Q}$ and 3rd TRAINING performed after 5 weeks ( 1 week of training +4 weeks being left undisturbed in their home cage) did not influence performance in the memory acquisition of AAPAT in the group of young rats to 2ndTRAINING (Figure 5B III-5F III). Rats presented similar performance of the avoidance on each day of 2nd and 3rd TRAINING, whereas the effect of days was significant for comparisons between 1st and 3rd TRAINING (Supplementary Data 9).

\section{DISCUSSION}

The Geroscience Hypothesis asserts that the same molecular and cellular mechanisms are central to aging and age-related disorders [29]. Accumulation of senescent cells is considered as one of the key processes responsible for the functional deterioration in aging. A growing body of literature suggests that senolytic treatment may alleviate multiple geriatric syndromes and diseases occurring in advanced age [20]. Only recently the improvement of memory and learning skills upon clearance of senescent cells with the combination of $\mathrm{D}+\mathrm{Q}$ in aged mice has been reported [25]. In the present study we show that 8 weeks of $D+Q$ administration alleviated cognitive impairments associated with aging in Wistar rats. Aged rats following $\mathrm{D}+\mathrm{Q}$ treatment displayed improved learning abilities and effective acquisition of spatial short- and long-term memory of a new place compared to vehicletreated rats. Our results complement and extend previous reports on the beneficial effects of senolytics in treatment of age-related disorders. Previous data on $\mathrm{D}+\mathrm{Q}$ action in aging has included mouse models (reviewed in [16], therefore to our knowledge, this is the first report describing the use of those drugs in rats, in such a context. Rats could be considered a very useful animal model for studying aging-related brain conditions as they exhibit a significant age-dependent decrease in cognitive abilities [30]. Moreover, significantly elevated inflammation baseline level has been observed in aged rats [31]. Most importantly, rats are considered to be particularly suitable for measuring cognitive abilities in spatial tasks due to their natural affinity to interactions with spatial and social environments [32]. All the above arguments prompted us to use rats for the present research. We used outbred stock Wistar rats because, in our opinion, this animal model better represents the heterogeneity among older adults in the rate of decline of perceptual reasoning and processing speed. Aging is associated with significant heterogeneity in cognitive and noncognitive behavioral abilities among both laboratory animals and humans $[33,34]$. By testing the same cohort of aged animals before and after $\mathrm{D}+\mathrm{Q}$ treatment in the same spatial task but to the place described by the new spatial coordinates 


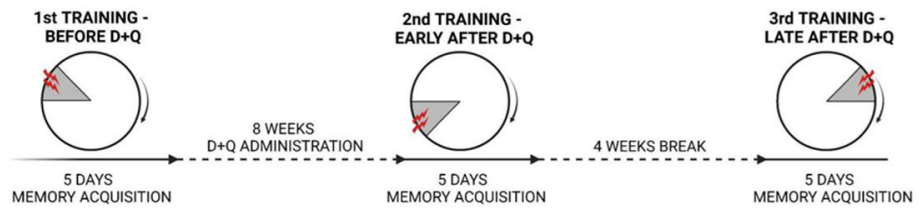

$1^{\text {st }}$ TRAINING vs $2^{\text {nd }}$ TRAINING

$1^{\text {st }}$ TRAINING vs $3^{\text {rd }}$ TRAINING

$2^{\text {nd }}$ TRAINING vs $3^{\text {rd }}$ TRAINING
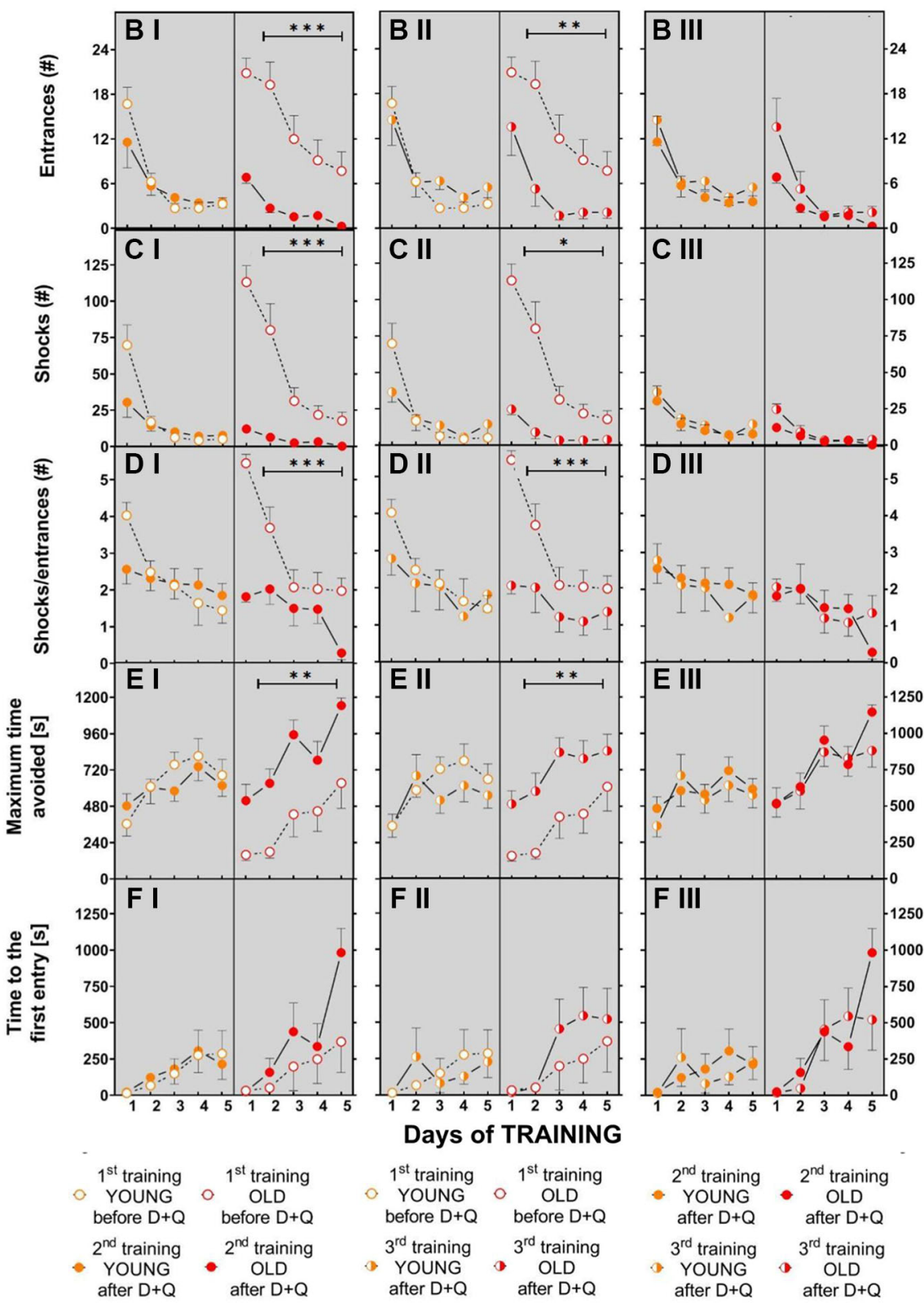

Days of TRAINING
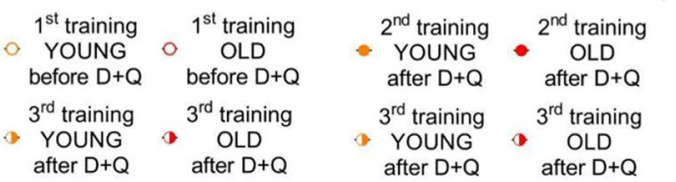

Figure 5. Treatment with $D+Q$ significantly improves cognitive abilities in aged rats in spatial memory task in the AAPAT on spatial memory for at least $\mathbf{5}$ weeks after treatment termination. (A) Schematic diagram of the experimental design in which rats are subjected to the training 3 times, each time with a new position of the to-be-avoided place. The number of entries into the shock place (\# of entries, B I-III), the number of shocks (\# shocks, C I-III) and number of shocks per one entry (Shocks/entrance, D I-III) together with maximum time avoided (E I-III) and time to the first entry (F I-III) during place avoidance training reflect changes in the learning abilities and memory in aged rats not only early after $D+Q$ treatment (2nd TRAINING) but also after additional 4 weeks (3rd TRAINING). Performance of place avoidance by young (3-month-old; YOUNG; orange color) and aged (18-month-old; OLD; red color) rats in the AAPAT before and after 8 weeks of treatment with $D+Q$ by oral gavage. Figures labeled with "I" depict comparison between 1st TRAINING and 2nd TRAINING, "II" between 1st TRAINING and 3rd TRAINING, whereas "III" depict comparison between 2nd TRAINING and 3rd TRAINING. The results are expressed as mean $\pm \mathrm{SEM}, N=7,{ }^{*} p<0.05,{ }^{* *} p<0.01,{ }^{* * *} p<0.001$. Data obtained for groups at consecutive trials were compared using repeated measures two-way ANOVA. 
we could limit the effect of initial heterogeneity in our data. In the active allothetic place avoidance task rats learn to avoid the virtual place of the rotating arena where short-lasting, weak, sporadic electrical shocks are administered. This place is in a fixed position to the distal room cues, whereas the proximal cues from the arena and from the movement are misleading what requires stimuli segregation and engaging cognitive coordination processes $[26,35]$. This makes the test more challenging compared to the commonly used test for studying spatial memory, such as the Morris water maze test, where proximal, misleading stimuli, e.g. defecation, urination, which could confuse spatial orientation are dissolved in water. It has been shown previously, by testing young-adult rats using the AAPAT, that spatial memory, which demands cognitive coordination, was very sensitive to different treatments, e.g. tetrodotoxin (TTX) unilateral hippocampal reversible blockade [36], application of NMDA antagonists such as memantine or MK-801 that cause memory dysfunctions [37], or silver nanoparticles [38]. We found AAPAT to be a complex and sensitive method to measure cognitive skills, particularly in aged rats, since it can be completed despite age-associated with a decrease in physical fitness, which could be a confounding factor in tests requiring physical effort to complete such as Morris water maze test.

One of the primary modes of action of senolytics is the clearance of senescent cells, which release mediators of inflammation such as cytokines, chemokines and growth factors. The decreasing burden of senescent cells has been shown to reduce peripheral inflammation [27]. Indeed, similarly to previously published results from mouse models, we show that aged but not young rats respond to senolytic treatment with a significant drop in the levels of serum factors associated with SASP. Even though removal of senescent cells from different organs and tissues by $\mathrm{D}+\mathrm{Q}$ has been a well-described phenomenon there is relatively small literature on that subject for the central nervous system in normal aging. Among those reports, some data provide insight into the senolytic activity of $\mathrm{D}+\mathrm{Q}$ in the brain. Tau-containing neurofibrillary tangles (NFT) that accumulate in the brains of Alzheimer's Disease (AD) patients and mice models of neurodegeneration display cellular senescence-like transcriptomic profiles. Magnetic Resonance Imaging (MRI) and histopathological analyses of the brains from $\mathrm{D}+\mathrm{Q}$ treated $\mathrm{rTg}(\operatorname{tauP} 301 \mathrm{~L}) 4510$ transgenic mice showed reduction in the total Tau-containing neurofibrillary tangles (NFT) density what was associated with increased levels of synaptophysin and PSD 95 proteins expression [39]. Bussian et al. found that p16-positive astrocytes and microglia accumulate in the brains of mice model of tau- dependent neurodegenerative disease. By genetic or pharmacological clearance of these cells they managed to prevent gliosis, NFT deposition, degeneration of neurons and cognitive function deterioration [40]. In another study, in the brains of patients with $\mathrm{AD}$ and $\mathrm{AD}$ mouse models, A $\beta$ plaque-associated oligodendrocyte progenitor cells but not astrocytes, microglia, or oligodendrocytes, exhibited a senescence-like phenotype characterized by the upregulation of $\mathrm{p} 21, \mathrm{p} 16$ protein expression, and senescence-associated-betagalactosidase (SA- $\beta$-gal) activity. Senolytic treatment of $\mathrm{AD}$ mice selectively removed senescent cells from the plaque environment, reduced neuroinflammation, decreased $A \beta$ load, and ameliorated cognitive deficits [23]. In an interesting paper by Ogrodnik et al., alleviation of anxiety symptoms in the high-fat diet fed mice was achieved by intra gavage treatment with $\mathrm{D}+\mathrm{Q}$ [41]. In the recent study Ogrodnik et al. revealed ageassociated increase in the percentage of p16-positive microglia and oligodendrocyte progenitor cells in the hippocampi of aged mice. Although whole body genetic elimination of p16-positive senescent cells resulted in similar improvement of learning and memory measured in stone $\mathrm{T}$ maze compared to $\mathrm{D}+\mathrm{Q}$ treatment, the reduction of p16 was not observed in the microglial population of the hippocampus in D + Q-treated mice [25]. Those results suggest that the beneficial effects of $\mathrm{D}+\mathrm{Q}$ treatment might be mediated by the decrease of senescence burden in the periphery rather than in the central nervous system. From the above cited studies emerges differential susceptibility of various brain regions and cell types to the treatment-dependent attenuation of senescence. This could partially explain why we were not able to detect the downregulation of cell senescence markers (Supplementary Figures 3 and 4). In depth analysis using more sensitive methods and better defined brain regions and cell types potentially could allow detection of the changes in the levels of the senescence markers upon $\mathrm{D}+\mathrm{Q}$ treatment. Those changes could translate into behavioral effects since a relatively small number of senescent cells in a given tissue may profoundly contribute to the spreading of low grade inflammation [42] which in aged animals participate in the inflammaging [12]. Inflammaging, in turn, is connected with cognitive impairment, what was reported not only for animals, but also for humans [43]. Thus, reduction of the low grade inflammation state observed by us in the serum of $\mathrm{D}+\mathrm{Q}$ treated animals may as well have both direct and indirect effects on the brain function via changes in the cardiovascular function [44]. Further studies are necessary to estimate the extent to which $\mathrm{D}+\mathrm{Q}$ improves memory and learning skills by directly affecting the brain tissue in aged animals.

Storing and processing information in the neural network depends on the number and quality of 
functional connections in the brain. Hippocampus, a brain structure critical for learning and memory formation, is particularly susceptible to aging-associated disorders such as Alzheimer's Disease [45]. Overall, the hippocampus retains its architecture during aging, however functional alterations, such as impairment of long-term potentiation (LTP) induction or maintenance are reflected in aberrant dendritic spine morphology and gene expression. Long and thin dendritic spines seem to be particularly susceptible to aging. Those spines create new synaptic connections making it possible for the neuronal network to encode new information [46]. We found that $\mathrm{D}+\mathrm{Q}$ treatment shifts the spine morphology of CA1 hippocampal neurons towards less mature connections which may explain the improved ability of aged rats to learn how to avoid the shock place in the AAPAT. Interestingly, we found that these changes of dendritic spine morphology are exclusive to the apical dendrites and not basal dendrites. Basal and apical dendrites of the CAlhippocampal region, despite being branches of the same pyramidal cells, have distinct protein composition, biophysical properties and are characterized by different inputs [47]. However, to the best of our knowledge, little is known about the impact of aging on those two portions of the dendritic tree. One study revealed aberrant LTP in basal but not apical dendrites of hippocampal field CA1 in slices prepared from middle aged 7- to 10-month-old rats compared to 3-month-old young adults [48]. The relatively young age of animals and lack of morphological data makes it difficult to extrapolate it to the data presented here.

Dynamic plasticity of adult neurons requires specific, strictly regulated and rapid changes in the gene expression. One of the mechanisms that has been implicated in the regulation of expression of genes associated with synaptic plasticity is histone $\mathrm{H} 3$ methylation. In particular trimethylation of H3K27 and H3K9. Even though both are associated with transcription repression, multiple studies have shown age-associated downregulation of $\mathrm{H} 3 \mathrm{~K} 27 \mathrm{me} 3$ and upregulation of H3K9me3 in brain tissue. Significant drop in H3K27me3 levels found in brains of 22-monthold $\mathrm{BALB} / \mathrm{c}$ mice compared to 3-month-old animals is reversed by caloric restriction, a treatment that improves cognitive abilities in aged animals [8]. On the other hand, in young mice, elevated levels of $\mathrm{H} 3 \mathrm{~K} 9 \mathrm{me} 3$ occurring in postoperative recovery after isoflurane anesthesia cause cognitive impairments in fear conditioning paradigm [49]. Accordingly, downregulation of $\mathrm{H} 3 \mathrm{~K} 9 \mathrm{me} 3$ in the hippocampus by inhibition of SUV39H1 leads to memory improvements in aged mice. Interestingly, this downregulation of $\mathrm{H} 3 \mathrm{~K} 9 \mathrm{me} 3$ is accompanied by an increase in both thin and stubby spine in the CA1 region only at apical and not at the basal dendritic tree, which stands in agreement with our findings [9]. Strikingly similar results concerning changes in histone methylation profile have been obtained in the acute and subchronic stress rat models. Rats restrained once or for 7 days (30 min/day) displayed reduced levels of $\mathrm{H} 3 \mathrm{~K} 27 \mathrm{me} 3$ and increased basal levels of $\mathrm{H} 3 \mathrm{~K} 9 \mathrm{me} 3$ in the hippocampus [50]. Since a plethora of published data have linked stress with aberrant synaptic plasticity, it is plausible that those two repressive marks play an important role in the regulation of neuronal connectivity [51]. One potential mechanism linking brain histone $\mathrm{H} 3$ trimethylation and synaptic plasticity is BDNF, a potent regulator of synaptic plasticity and neuronal activity. Changes in the BDNF expression have been shown to inversely correspond to increased $\mathrm{H} 3 \mathrm{~K} 9 \mathrm{me} 3$ in a postoperative model of cognitive impairment [49] or downregulation in mice with inhibited activity of SUV39H1 [9]. Moreover, gavage administration of quercetin at doses of 20 and 50 $\mathrm{mg} / \mathrm{kg}$ has been shown to result in a significant increase in the hippocampal BDNF mRNA [52]. Further studies are necessary to fully understand the role of histone methylation in the beneficial effects of $\mathrm{D}+\mathrm{Q}$ on cognitive impairment in aged animals.

In summary, we found that $\mathrm{D}+\mathrm{Q}$ treatment alleviates cognitive behavioral deficits in aged but not young Wistar male rats in the AAPAT, which is accompanied by decreased peripheral inflammation measured by serum concentration of cytokines and growth factors (including SASP factors). Despite the lack of evidence of decreased senescent cell burden in the brain tissue, we found that improvement in learning and memory coincides with changes at the synaptic plasticity level. Dendritic spines on the apical dendrites of CA1 neurons of the hippocampus become longer and less mature, which we believe translates into more efficient formation of new connections. Since connectivity between neurons is tightly regulated at the molecular level, any changes in that process require alterations in the gene expression. Indeed, we found that, upon $\mathrm{D}+\mathrm{Q}$ treatment, methylation of histone $\mathrm{H} 3$, at the lysines residues 9 and 27, which has been shown to play an important role in age-associated cognitive decline, is modified in aged rats compared to vehicle treated groups and it is similar to that seen in young animals. Our findings provide mechanistic insight into the beneficial effects of $\mathrm{D}+\mathrm{Q}$ on cognitive abilities in advanced age that may contribute to future studies associated with treatment of aging-associated diseases.

\section{MATERIALS AND METHODS}

\section{Animals}

Male Wistar 3-month-old rats $(n=23)$, 18-month-old rats $(n=7)$ and 22-month-old $(n=16)$ were obtained from the animal house of the Mossakowski Medical 
Research Center, Polish Academy of Sciences (Warsaw, Poland) or from Janvier Labs (Le Genest-Saint Isle, France). Animals were housed 3-4 per cage under a $12 \mathrm{~h} / 12 \mathrm{~h}$ light/dark cycle (lights on at 8:00 AM) with food and water available ad libitum. In the present study we used only male animals due to ready availability of aged rats of that sex.

\section{Experiment design}

The experiment consisted of analyzing behavior of animals in the Active Allothetic Place Avoidance Task (AAPAT). The experiments were performed on two groups of animals. The first group (GROUP 1) consisted of $n=16$ young (3-month-old rats at the beginning of the experiment) and $n=16$ aged (22month-old rats at the beginning of the experiment). Young and aged animals were divided into groups of 8 rats which were later assigned to $\mathrm{D}+\mathrm{Q}$ or vehicle treatment. Animals were tested 2 times in the AAPAT: before $\mathrm{D}+\mathrm{Q}$ or vehicle treatment (1st TRAINING) and directly after cessation of $\mathrm{D}+\mathrm{Q}$ treatment (2nd TRAINING). $87 \%$ (7 out of 8) animals in each treatment cohort survived until the end of the experiment. The second group (GROUP 2) used in order to check the long-term effects of $\mathrm{D}+\mathrm{Q}$ on learning and memory formation consisted of $n=7$ aged (18month-old rats at the beginning of the experiment) and $n=7$ young (3-month-old rats at the beginning of the experiment) which were tested 3 times in the AAPAT: before $\mathrm{D}+\mathrm{Q}$ or vehicle treatment (1st TRAINING), directly after cessation of $\mathrm{D}+\mathrm{Q}$ treatment (2nd TRAINING) and 5 weeks after the treatment discontinuation (4 weeks past 2nd TRAINING end - 3rd TRAINING). $100 \%$ (7 out of 7 ) animals in each treatment cohort survived until the end of the experiment. Due to injuries, results from one aged rat from GROUP 1 (D+Q treatment cohort, 2nd training) and results of one young rat from GROUP $2(D+Q$ treatment cohort, 3rd training) were either not recorded or excluded from the final analysis after confirming impaired basic locomotion in the open field test. The schematic representation of experimental designs for each group are depicted in Figure 1A and Figure 5A, respectively. Before spatial memory acquisition training, all animals were habituated to the arena by placing them on the still arena without shock delivery for 10 min daily. After 5 days of habituation, spatial memory acquisition training in AAPAT began, with one 20 min session daily for 5 days. During the 1 st TRAINING shocks were delivered within a virtual sector of the arena according to room-frame coordinates (northwest). After the 1st TRAINING, the rats received $5 \mathrm{mg} / \mathrm{kg}$ dasatinib (LC Laboratories) plus $50 \mathrm{mg} / \mathrm{kg}$ quercetin (Sigma) dissolved in 60\% Phosal, 10\% ethanol, and 30\% PEG-400 or solvent (vehicle) alone
(60\% Phosal, $10 \%$ ethanol, and $30 \%$ PEG-400) in equivalent volume. The $\mathrm{D}+\mathrm{Q}$ solution or vehicle was administered by oral gavage for 5 days per week every 8 weeks. After D+Q treatment, 2nd TRAINING of active place avoidance began, with a new position of the shock place (i.e., southwest). After the 2nd TRAINING, GROUP 2 was left undisturbed for another 4 weeks. After this 4-week period, AAPAT was conducted again (3rd TRAINING), this time with a shock place at the new position (i.e., northeast). The body mass of the animals was monitored throughout the experiment. At the end of 2nd TRAINING in group first Open Field Test were performed. Animals were euthanized on the next day after the last behavioral test.

\section{Analysis of cytokines and growth factors in blood serum}

Arterial blood was collected in tubes containing a clot activator from heart puncture at the end of the study. Within an hour, the blood was centrifuged, aliquoted and stored at $-80^{\circ} \mathrm{C}$. Serum was analyzed by Eve Technologies (Calgary, Canada) using the Multiplex assay (Millipore) in two technical replicates. The following cytokines and growth factors were analyzed: tumor necrosis factor alpha (TNF $\alpha)$, interleukin-1 alpha IL-1 $\alpha$ ), IL-1 $\beta$, IL-2, IL-4, IL-5, IL-6, IL-18, IL10, IL-12, IL-13, IL-17A, monocyte chemoattractant protein-1 (MCP-1), macrophage inflammatory protein 1-alpha (MIP-1 $\alpha$ ), MIP-2, regulated upon activation, normal T-cell expressed and secreted (RANTES), C$\mathrm{X}-\mathrm{C}$ motif chemokine 5 (LIX), fractalkine, interferon gamma-induced protein 10 (IP-10), leptin, eotaxin, interferon $\gamma$ (IFN $\gamma)$, granulocyte-colony stimulating factor (G-CSF), granulocyte-macrophage colonystimulating factor GM-CSF, human growth-regulated oncogene/keratinocyte chemoattractant (GRO/KC), and vascular endothelial growth factor (VEGF). Out of range values were randomly sampled from the range between 0 and the lowest value of the standard curve used to calculate serum factors concentrations. For statistical analysis we used $\log 2$ of raw values normalized to the average of the young vehicle group. Bar plots for each of the analytes can be found in Supplementary Figure 2.

\section{DiI staining of brain slices and synaptic plasticity analysis}

To visualize changes in the shape of dendritic spines, 1,1'-dioctadecyl-3,3,3,3'-tetramethylindocarbocyanine perchlorate (DiI) staining was performed on hippocampal sections. The rat brain tissue was fixed in $4 \%$ PFA. The brains were dissected and sliced using a vibratome. Slices $(140 \mu \mathrm{m}$ thick) were allowed to recover for at least $1.5 \mathrm{~h}$ at room temperature in PBS. 
Random dendrite labeling was performed using $1.6 \mu \mathrm{m}$ tungsten particles (Bio-Rad, Hercules, CA, USA) that were coated with propelled lipophilic fluorescent dye (DiI; Invitrogen) and was delivered to the cells by gene gun (Bio-Rad) bombardment. Images of dendrites in the hippocampus were acquired under $561 \mathrm{~nm}$ fluorescent illumination using a confocal microscope $(63 \times$ objective, 1.4 NA) at a pixel resolution of $1024 \times 1024$ with a 3.43 zoom, resulting in a $0.07 \mu \mathrm{m}$ pixel size. The images that were acquired from the brain slices were processed using ImageJ software (National Institutes of Health, Bethesda, MD, USA) and analyzed semiautomatically using custom-written SpineMagick software (patent no. WO/2013/021001). The analyzed dendritic spines belonged to secondary and ternary dendrites to reduce possible differences in spine morphology that are caused by the location of spines on dendrites with different ranks. Moreover, spontaneous changes in dendritic spine shape can obscure systematic effects because of the spontaneous intrinsic fluctuation of dendritic spine shape. To minimize this effect in the analysis, we used a scale-free parameter of relative changes in the spine length-to-head width ratio, which reflects spine shape. The spine length was determined by measuring the curvilinear length along a fitted virtual skeleton of the spine. The fitting procedure was performed by looking for a curve along which integrated fluorescence was at a maximum. The head width was defined as the diameter of the largest spine section while excluding the bottom part of the spine ( $1 / 3$ of the spine length adjacent to the dendrite). Dendritic segments of at least 3 animals per condition were morphologically analyzed resulting in 4400 (VEH) and $6200(\mathrm{D}+\mathrm{Q})$ spines. To determine spine density, approximately $900(\mathrm{VEH})$ and $1500(\mathrm{D}+\mathrm{Q}) \mu \mathrm{m}$ of dendritic length was analyzed per experimental group.

\section{Histone isolation, Western blotting and quantification}

Histones were isolated from frozen hippocampi. First, the tissue was homogenized in lysis buffer $(250 \mathrm{mM}$ sucrose, $50 \mathrm{mM}$ TrisHCl, $\mathrm{pH} 7.5,25 \mathrm{mM} \mathrm{KCl}$ with protease (Roche 04693159001) and phosphatase (Roche 04906837001) inhibitors (added immediately prior to use) using glass homogenizer (15-20 strokes). Homogenate was then centrifuged at $7000 \mathrm{~g}$ for $1 \mathrm{~min}$ and the pellet containing nuclear fraction was resuspended in an extraction buffer $(0.5 \mathrm{~N} \mathrm{HCl}, 10 \%$ glycerol). After $1 \mathrm{~h}$, samples were centrifuged at 12100 $\mathrm{g}$ for $5 \mathrm{~min}$. Histones were precipitated from the supernatant with acetone and pelleted by centrifugation at $12100 \mathrm{~g}$ for $5 \mathrm{~min}$. Dried pellet was resuspended in $50 \mathrm{mM}$ TrisHCl with 3\% SDS. Isolated protein concentration was determined by BCA assay. The isolated histone fractions were then denatured in a sample buffer heated to $95^{\circ} \mathrm{C}$ for $5 \mathrm{~min}$. About $10 \mathrm{ug}$ of histone fractions were separated by a $15 \%(\mathrm{w} / \mathrm{v})$ SDSPAGE and transferred onto a nitrocellulose membrane (Amersham GE Healthcare). After blocking the membrane with $5 \%(\mathrm{w} / \mathrm{v})$ non-fat milk in Tris-Buffered Saline Tween-20 (TBST), the membrane was incubated overnight with primary antibody, washed in TBST, incubated with Horseradish peroxidase (HRP) conjugated secondary antibody (anti-mouse and anti-rabbit, Dako Denmark $\mathrm{A} / \mathrm{S}$ ) at 1:2000 dilution in milk, washed and developed with ECL (Thermo Fisher Scientific). To obtain total $\mathrm{H} 3$ the membranes were stripped with a fresh stripping buffer $(1.5 \%$ glycine, $0.1 \%$ SDS, $1 \%$ Tween, $\mathrm{HCl}, \mathrm{pH} 2.2$ ) for $20 \mathrm{~min}$, washed twice with PBS and twice with TBST. Then, the blotting was conducted as described above. The following primary antibodies were used: H3 (Abcam ab1791, 1:4000 dilution), H3K27me3 (Diagenode C15410195, 1:1000 dilution), H3K9me3 (Diagenode C15410193, 1:1000 dilution). Developed films were scanned and signal intensities were quantified using Image $\mathrm{J}$ (National Institutes of Health, Bethesda, MD, USA).

\section{Active allothetic place avoidance test (AAPAT) - detailed description of the method}

The place avoidance apparatus was described in detail in previous publications $[26,36]$. The apparatus consisted of an 80-cm-diameter, elevated $(80 \mathrm{~cm})$, rotating $(1 \mathrm{rpm})$ metal arena rimmed by a metal lip on the periphery $(2 \mathrm{~cm})$. The arena was located in the center of a $3 \mathrm{~m} \times 4 \mathrm{~m}$ room with dim light $( \pm 241 \times)$ and explicit visual landmarks. Infrared light-emitting diodes (LED) were attached to the latex harness on the rat's back. A computer system tracked the light-emitting diode position in the reference frame of the room every $20 \mathrm{~ms}$ by using an infrared-sensitive TV camera. The room frame position of a second LED fixed to the periphery of the arena also was tracked and was used to calculate the rat's position in the reference frame of the arena. Thus, a to-be-avoided sector was defined in both room and arena reference frame. Before the experiment, rats were implanted with a 25-gauge $(0.50 \mathrm{~mm})$ hypodermic needle, puncturing the skin fold on their backs. The sharp end of the needle was then cut off and a small loop was formed with tweezers. This loop was connected by a mini-alligator clip that was attached with a cable to a shock box that was used for the delivery of electric shocks. Every time the rat entered the to-be-avoided sector (60 degrees), the computer triggered a mild, constant current $(50 \mathrm{~Hz}, 0.5 \mathrm{~s})$ footshock that was delivered across the low- and high impedance electrodes. The low-impedance $(\sim 100 \Omega)$ shock electrode was clipped to the needle loop on the back of the rat, while the high-impedance $(\sim 100 \mathrm{~K} \Omega)$ electrode was produced by contact of the rat's feet to the grounded arena surface. The intensity of current was 
individually adjusted for each animal to provoke an escape reaction, ranging between 0.2 and $0.3 \mathrm{~mA}(50$ $\mathrm{Hz}$ ). If the rat did not leave the sector, the shock was repeated every $1.5 \mathrm{~s}$. Commercial software was used for data collection and analysis (BioSignal Group, USA).

The AAPAT is a variant of the original place avoidance test (Bures et al., 1997). In the place avoidance task, locating the shock sector requires place navigation ability, and the execution of avoidance requires an instrumental response. Compared with standard spatial memory tests, the AAPAT is unique because the formation of spatial representation of a to-be-avoided place on a rotating arena requires ongoing segregation of room relevant stimuli from misleading. Spatial memory in the AAPAT was evaluated based on such parameters as: number of entrances, number of shocks on the shocks place, maximum time avoided and time to the first entrance into the shock place. Reduction of the number of entries (errors) and a reduction of the number of short-lasting shocks that were repeated until the rat escaped the shock place described effective acquisition of spatial memory, whereas long maximum time avoided expressed functioning of short-term memory. The increase in the time to the first entry into the shock place reflected the formation of long-term memory traces on consecutive training days.

\section{Open-field test}

All open-field testing took place inside a close, well lighted room with minimal sound noise. The open-field arena $(100 \mathrm{~cm}(\mathrm{l}) 100 \mathrm{~cm}(\mathrm{w}) 40 \mathrm{~cm}(\mathrm{~h}))$ consisted of four opaque walls and a black matte floor. Prior to testing each animal, the entire open-field arena was cleaned using $70 \%$ alcohol. All animals were tested in the same manner, in brief, every animal was carefully placed near the same corner facing the wall. Video recording started prior to depositing the animal in the arena while analysis of the video footage started 15 seconds after. This was done to allow the experimenter time to leave the room and the animal to resume its behavior following the closing of the door. Video footage was analyzed using Toxtrac (https://source forge.net/projects/toxtrac). Parameters include det.mins $=3000$, det.maxs $=50000$, det.thr $1=150$, det.thr $2=$ 240. For exploration rate, the exploration was set as $100 \mathrm{~mm}$ in all edge settings which allows for dividing the whole arena in $10 \mathrm{~cm}^{2}$ squares. Every time the animal entered one square it counted as one explored square.

\section{SA- $\beta$-galactosidase activity}

PFA fixed brains of either vehicle or $\mathrm{D}+\mathrm{Q}$ treated rats were cut into $30 \mu \mathrm{m}$ slices. Hippocampi were isolated and SA- $\beta$-galactosidase activity was measured. Slices were fixed in a buffer containing unmoved formaldehyde and $8 \%$ glutaraldehyde in PBS for 10 minutes, rinsed three times in PBS and incubated with $\mathrm{x}$-gal buffer at $37^{\circ} \mathrm{C}$ in an air-tight box for $8 \mathrm{~h}$. After SA- $\beta$-galactosidase detection, slices were processed for immunofluorescence.

Astrocytes and neurons were targeted by immunofluorescence prior to SA- $\beta$-galactosidase staining with either anti-GFAP or anti-NeuN antibodies to define regions of interest by extracting the sum projection of either staining after thresholding the signal intensity using Fiji (ImageJ) software (National Institutes of Health, Bethesda, MD, USA). Within each region of interest, mean grey value of SA- $\beta$-galactosidase intensity was measured using the same software.

\section{Statistical analysis}

Two compare data pairs we used Mann-Whitney test as a nonparametric, Student's $t$ test for parametric data. Nested $t$-test was used for the analysis of spine morphology. For analysis of AAPAT 5 day training curves or serum cytokine profiles between VEH and $\mathrm{D}+\mathrm{Q}$ within one age group we used two-way ANOVAs followed by Tukey post hoc test. For analysis of cytokine profiles between young and aged rats we used two-way ANOVAs followed by Tukey post hoc test. $P$ values lower than 0.05 were considered significant. All statistical tests were performed using GraphPad Prism 8 (GraphPad, San Diego, CA, USA). Data are presented as mean $+/-$ SEM.

\section{Data availability statement}

Data available on request from the authors.

\section{AUTHOR CONTRIBUTIONS}

A.K., M.W., E.S., J.W., M.D., G.M., A.B-Z., A.G., I.F., G.P., conceived and designed the study. A.K. and M.W. wrote the manuscript. A.K., M.W., G.P., A.G., M.D. performed the experimental works and analyzed the data. E.S., J.W., M.D., G.M., A.B-Z., A.G., G.P., I.F., edited the manuscript.

\section{CONFLICTS OF INTEREST}

The authors declare no conflicts of interest related to this study.

\section{FUNDING}

This work was supported by the by the National Science Center grants: UMO-2017/26/E/NZ4/00637 (JW, AK), UMO-2019/35/B/NZ4/01920 (ES, AK, MW, ES, JW, MD, GM, AB-Z, IF, GP). 


\section{REFERENCES}

1. Marois G, Muttarak R, Scherbov S. Assessing the potential impact of COVID-19 on life expectancy. PLoS One. 2020; 15:e0238678.

https://doi.org/10.1371/journal.pone.0238678 PMID: $\underline{32941467}$

2. Harada CN, Natelson Love MC, Triebel KL. Normal cognitive aging. Clin Geriatr Med. 2013; 29:737-52. https://doi.org/10.1016/j.cger.2013.07.002 PMID:

3. West MJ, Coleman PD, Flood DG, Troncoso JC. Differences in the pattern of hippocampal neuronal loss in normal ageing and Alzheimer's disease. Lancet. 1994; 344:769-72. https://doi.org/10.1016/s0140-6736(94)92338-8 PMID: 7916070

4. Adams MM, Donohue HS, Linville MC, Iversen EA, Newton IG, Brunso-Bechtold JK. Age-related synapse loss in hippocampal CA3 is not reversed by caloric restriction. Neuroscience. 2010; 171:373-82. https://doi.org/10.1016/j.neuroscience.2010.09.022 PMID:20854882

5. Bloss EB, Janssen WG, Ohm DT, Yuk FJ, Wadsworth S, Saardi KM, McEwen BS, Morrison JH. Evidence for reduced experience-dependent dendritic spine plasticity in the aging prefrontal cortex. J Neurosci. 2011; 31:7831-9. https://doi.org/10.1523/JNEUROSCI.0839-11.2011 PMID:21613496

6. Parkel S, Lopez-Atalaya JP, Barco A. Histone H3 lysine methylation in cognition and intellectual disability disorders. Learn Mem. 2013; 20:570-9.

https://doi.org/10.1101/Im.029363.112 PMID:24045506

7. Kushwaha A, Thakur MK. Increase in hippocampal histone H3K9me3 is negatively correlated with memory in old male mice. Biogerontology. 2020; 21:175-89.

https://doi.org/10.1007/s10522-019-09850-1 PMID:31760560

8. Gong H, Qian H, Ertl R, Astle CM, Wang GG, Harrison $\mathrm{DE}, \mathrm{XU} \mathrm{X}$. Histone modifications change with age, dietary restriction and rapamycin treatment in mouse brain. Oncotarget. 2015; 6:15882-90.

https://doi.org/10.18632/oncotarget.4137 PMID:26021816

9. Snigdha S, Prieto GA, Petrosyan A, Loertscher BM, Dieskau AP, Overman LE, Cotman CW. H3K9me3 Inhibition Improves Memory, Promotes Spine Formation, and Increases BDNF Levels in the Aged Hippocampus. J Neurosci. 2016; 36:3611-22.
https://doi.org/10.1523/JNEUROSCI.2693-15.2016 PMID:27013689

10. Sikora E, Bielak-Zmijewska A, Dudkowska M, Krzystyniak A, Mosieniak G, Wesierska M, Wlodarczyk J. Cellular Senescence in Brain Aging. Front Aging Neurosci. 2021; 13:646924.

https://doi.org/10.3389/fnagi.2021.646924

PMID: 33732142

11. Freund A, Orjalo AV, Desprez PY, Campisi J. Inflammatory networks during cellular senescence: causes and consequences. Trends Mol Med. 2010; 16:238-46.

https://doi.org/10.1016/i.molmed.2010.03.003 PMID:20444648

12. Franceschi C, Campisi J. Chronic inflammation (inflammaging) and its potential contribution to ageassociated diseases. J Gerontol A Biol Sci Med Sci. 2014 (Suppl 1); 69:S4-9.

https://doi.org/10.1093/gerona/glu057 PMID:24833586

13. Avital A, Goshen I, Kamsler A, Segal M, Iverfeldt K, Richter-Levin G, Yirmiya R. Impaired interleukin-1 signaling is associated with deficits in hippocampal memory processes and neural plasticity. Hippocampus. 2003; 13:826-34. https://doi.org/10.1002/hipo.10135 PMID:14620878

14. Balschun D, Wetzel W, Del Rey A, Pitossi F, Schneider $\mathrm{H}$, Zuschratter W, Besedovsky HO. Interleukin-6: a cytokine to forget. FASEB J. 2004; 18:1788-90.

https://doi.org/10.1096/fj.04-1625fje PMID: 15345694

15. Kirkland JL, Tchkonia T, Zhu Y, Niedernhofer $L$, Robbins PD. The Clinical Potential of Senolytic Drugs. J Am Geriatr Soc. 2017; 65:2297-301.

https://doi.org/10.1111/jgs.14969

PMID:28869295

16. Sikora E, Bielak-Zmijewska A, Mosieniak G. Targeting normal and cancer senescent cells as a strategy of senotherapy. Ageing Res Rev. 2019; 55:100941.

https://doi.org/10.1016/j.arr.2019.100941 PMID:31408714

17. Zhu Y, Doornebal EJ, Pirtskhalava T, Giorgadze N, Wentworth M, Fuhrmann-Stroissnigg H, Niedernhofer L, Robbins PD, Tchkonia T, Kirkland JL. New agents that target senescent cells: the flavone, fisetin, and the BCL-X inhibitors, A1331852 and A1155463. Aging (Albany NY). 2017; 9:955-63. https://doi.org/10.18632/aging.101202 PMID:28273655

18. Short S, Fielder E, Miwa S, von Zglinicki T. Senolytics and senostatics as adjuvant tumour therapy. EBioMedicine. 2019; 41:683-92. 
https://doi.org/10.1016/j.ebiom.2019.01.056 PMID: $\underline{30737084}$

19. van Deursen JM. The role of senescent cells in ageing. Nature. 2014; 509:439-46.

https://doi.org/10.1038/nature13193 PMID:24848057

20. Wissler Gerdes EO, Misra A, Netto JME, Tchkonia T, Kirkland JL. Strategies for late phase preclinical and early clinical trials of senolytics. Mech Ageing Dev. 2021; 200:111591.

https://doi.org/10.1016/i.mad.2021.111591 PMID:34699859

21. Xu M, Pirtskhalava T, Farr JN, Weigand BM, Palmer $A K$, Weivoda MM, Inman CL, Ogrodnik MB, Hachfeld CM, Fraser DG, Onken JL, Johnson KO, Verzosa GC, et al. Senolytics improve physical function and increase lifespan in old age. Nat Med. 2018; 24:1246-56.

https://doi.org/10.1038/s41591-018-0092-9

PMID:29988130

22. Currais A, Farrokhi C, Dargusch R, Armando A, Quehenberger $O$, Schubert D, Maher P. Fisetin Reduces the Impact of Aging on Behavior and Physiology in the Rapidly Aging SAMP8 Mouse. J Gerontol A Biol Sci Med Sci. 2018; 73:299-307. https://doi.org/10.1093/gerona/g|x104 PMID:28575152

23. Zhang $P$, Kishimoto $Y$, Grammatikakis I, Gottimukkala K, Cutler RG, Zhang S, Abdelmohsen K, Bohr VA, Misra Sen J, Gorospe M, Mattson MP. Senolytic therapy alleviates $A \beta$-associated oligodendrocyte progenitor cell senescence and cognitive deficits in an Alzheimer's disease model. Nat Neurosci. 2019; 22:719-28.

https://doi.org/10.1038/s41593-019-0372-9

PMID: $\underline{30936558}$

24. Song S, Lam EW, Tchkonia T, Kirkland JL, Sun Y. Senescent Cells: Emerging Targets for Human Aging and Age-Related Diseases. Trends Biochem Sci. 2020; 45:578-92.

https://doi.org/10.1016/i.tibs.2020.03.008 PMID: 32531228

25. Ogrodnik $M$, Evans $S A$, Fielder E, Victorelli $S$, Kruger $P$, Salmonowicz $\mathrm{H}$, Weigand BM, Patel AD, Pirtskhalava T, Inman CL, Johnson KO, Dickinson SL, Rocha A, et al. Whole-body senescent cell clearance alleviates agerelated brain inflammation and cognitive impairment in mice. Aging Cell. 2021; 20:e13296.

https://doi.org/10.1111/acel.13296

PMID:33470505

26. Wesierska M, Dockery C, Fenton AA. Beyond memory, navigation, and inhibition: behavioral evidence for hippocampus-dependent cognitive coordination in the rat. J Neurosci. 2005; 25:2413-9. https://doi.org/10.1523/JNEUROSCI.3962-04.2005 PMID:15745968

27. Camell CD, Yousefzadeh MJ, Zhu Y, Prata LGP, Huggins MA, Pierson M, Zhang L, O'Kelly RD, Pirtskhalava T, Xun P, Ejima K, Xue A, Tripathi U, et al. Senolytics reduce coronavirus-related mortality in old mice. Science. 2021; 373:eabe4832.

https://doi.org/10.1126/science.abe4832 PMID:34103349

28. Coppé JP, Desprez PY, Krtolica A, Campisi J. The senescence-associated secretory phenotype: the dark side of tumor suppression. Annu Rev Pathol. 2010; 5:99-118.

https://doi.org/10.1146/annurev-pathol-121808102144

PMID:20078217

29. Kennedy BK, Berger SL, Brunet A, Campisi J, Cuervo AM, Epel ES, Franceschi C, Lithgow GJ, Morimoto RI, Pessin JE, Rando TA, Richardson A, Schadt EE, et al. Geroscience: linking aging to chronic disease. Cell. 2014; 159:709-13. https://doi.org/10.1016/j.cell.2014.10.039 PMID:25417146

30. Pepeu G. Mild cognitive impairment: animal models. Dialogues Clin Neurosci. 2004; 6:369-77. https://doi.org/10.31887/DCNS.2004.6.4/gpepeu PMID:22034045

31. Xin DL, Harris MY, Wade CK, Amin M, Barr AE, Barbe MF. Aging enhances serum cytokine response but not task-induced grip strength declines in a rat model of work-related musculoskeletal disorders. BMC Musculoskelet Disord. 2011; 12:63.

https://doi.org/10.1186/1471-2474-12-63

PMID:21447183

32. Hok V, Poucet B, Duvelle É, Save É, Sargolini F. Spatial cognition in mice and rats: similarities and differences in brain and behavior. Wiley Interdiscip Rev Cogn Sci. 2016; 7:406-21.

https://doi.org/10.1002/wcs.1411 PMID:27582415

33. Ardila A. Normal aging increases cognitive heterogeneity: analysis of dispersion in WAIS-III scores across age. Arch Clin Neuropsychol. 2007; 22:1003-11.

https://doi.org/10.1016/i.acn.2007.08.004 PMID: 17904332

34. Mota C, Taipa R, das Neves SP, Monteiro-Martins S, Monteiro S, Palha JA, Sousa N, Sousa JC, Cerqueira JJ. Structural and molecular correlates of cognitive aging in the rat. Sci Rep. 2019; 9:2005. 
https://doi.org/10.1038/s41598-019-39645-w PMID:30765864

35. Prokopova I, Bahnik S, Doulames V, Vales K, Petrasek T, Svoboda J, Stuchlik A. Synergistic effects of dopamine D2-like receptor antagonist sulpiride and $\beta$-blocker propranolol on learning in the carousel maze, a dry-land spatial navigation task. Pharmacol Biochem Behav. 2012; 102:151-6.

https://doi.org/10.1016/j.pbb.2012.04.003 PMID:22525744

36. Cimadevilla JM, Wesierska M, Fenton AA, Bures J. Inactivating one hippocampus impairs avoidance of a stable room-defined place during dissociation of arena cues from room cues by rotation of the arena. Proc Natl Acad Sci U S A. 2001; 98:3531-6. https://doi.org/10.1073/pnas.051628398 PMID: 11248112

37. Wesierska M, Svoboda J, Stuchlik A. A therapeutic dose of memantine improves the performance of rats in an active place avoidance task under the continuous dissociation of distal room and proximal arena cues. Neurobiol Learn Mem. 2019; 162:59-66. https://doi.org/10.1016/j.nlm.2019.03.011 PMID:31085330

38. Węsierska M, Dziendzikowska K, GromadzkaOstrowska J, Dudek J, Polkowska-Motrenko $\mathrm{H}$, Audinot JN, Gutleb AC, Lankoff A, Kruszewski M. Silver ions are responsible for memory impairment induced by oral administration of silver nanoparticles. Toxicol Lett. 2018; 290:133-44.

https://doi.org/10.1016/j.toxlet.2018.03.019 PMID:29578054

39. Musi N, Valentine JM, Sickora KR, Baeuerle E, Thompson CS, Shen Q, Orr ME. Tau protein aggregation is associated with cellular senescence in the brain. Aging Cell. 2018; 17:e12840.

https://doi.org/10.1111/acel.12840

PMID:30126037

40. Bussian TJ, Aziz A, Meyer CF, Swenson BL, van Deursen JM, Baker DJ. Clearance of senescent glial cells prevents tau-dependent pathology and cognitive decline. Nature. 2018; 562:578-82.

https://doi.org/10.1038/s41586-018-0543-y PMID:30232451

41. Ogrodnik $M$, Zhu $Y$, Langhi LGP, Tchkonia $T$, Krüger $P$, Fielder E, Victorelli S, Ruswhandi RA, Giorgadze N, Pirtskhalava T, Podgorni O, Enikolopov G, Johnson KO, et al. Obesity-Induced Cellular Senescence Drives Anxiety and Impairs Neurogenesis. Cell Metab. 2019; 29:1061-77.e8.

https://doi.org/10.1016/j.cmet.2018.12.008

PMID:
42. Biran A, Zada L, Abou Karam P, Vadai E, Roitman L, Ovadya Y, Porat Z, Krizhanovsky V. Quantitative identification of senescent cells in aging and disease. Aging Cell. 2017; 16:661-71.

https://doi.org/10.1111/acel.12592

PMID:28455874

43. Lin T, Liu GA, Perez E, Rainer RD, Febo M, CruzAlmeida $Y$, Ebner NC. Systemic Inflammation Mediates Age-Related Cognitive Deficits. Front Aging Neurosci. 2018; 10:236.

https://doi.org/10.3389/fnagi.2018.00236

PMID: 30127734

44. Tangestani Fard M, Stough C. A Review and Hypothesized Model of the Mechanisms That Underpin the Relationship Between Inflammation and Cognition in the Elderly. Front Aging Neurosci. 2019; 11:56.

https://doi.org/10.3389/fnagi.2019.00056

PMID:30930767

45. Bartsch $T$, Wulff $P$. The hippocampus in aging and disease: From plasticity to vulnerability. Neuroscience. 2015; 309:1-16. https://doi.org/10.1016/j.neuroscience.2015.07.084 PMID:26241337

46. Burke SN, Barnes CA. Neural plasticity in the ageing brain. Nat Rev Neurosci. 2006; 7:30-40. https://doi.org/10.1038/nrn1809 PMID: 16371948

47. Navakkode S, Sajikumar S, Korte M, Soong TW. Dopamine induces LTP differentially in apical and basal dendrites through BDNF and voltage-dependent calcium channels. Learn Mem. 2012; 19:294-9.

https://doi.org/10.1101//m.026203.112 PMID:22723051

48. Rex CS, Kramár EA, Colgin LL, Lin B, Gall CM, Lynch G. Long-term potentiation is impaired in middle-aged rats: regional specificity and reversal by adenosine receptor antagonists. J Neurosci. 2005; 25:5956-66. https://doi.org/10.1523/JNEUROSCI.0880-05.2005 PMID: 15976084

49. Wu T, Sun $X Y$, Yang $X$, Liu L, Tong $K$, Gao $Y$, Hao JR, Cao J, Gao C. Histone H3K9 Trimethylation Downregulates the Expression of Brain-Derived Neurotrophic Factor in the Dorsal Hippocampus and Impairs Memory Formation During Anaesthesia and Surgery. Front Mol Neurosci. 2019; 12:246. https://doi.org/10.3389/fnmol.2019.00246 PMID:31708739

50. Hunter RG, McCarthy KJ, Milne TA, Pfaff DW, McEwen BS. Regulation of hippocampal H3 histone methylation by acute and chronic stress. Proc Natl Acad Sci U S A. 2009; 106:20912-7. 
https://doi.org/10.1073/pnas.0911143106

PMID: 19934035

51. Qiao H, Li MX, Xu C, Chen HB, An SC, Ma XM. Dendritic Spines in Depression: What We Learned

from Animal Models. Neural Plast. 2016; 2016:8056370.

https://doi.org/10.1155/2016/8056370

PMID:26881133
52. Rahvar M, Owji AA, Mashayekhi FJ. Effect of quercetin on the brain-derived neurotrophic factor gene expression in the rat brain. Bratisl Lek Listy. 2018; 119:28-31.

https://doi.org/10.4149/BLL 2018006

PMID:29405727 


\section{SUPPLEMENTARY MATERIALS}

\section{Supplementary Data}

Aged rats presented worse memory acquisition in the place avoidance than young rats during the 1st TRAINING.

\section{Supplementary Data 1}

Aged non-treated rats assigned to vehicle or $\mathrm{D}+\mathrm{Q}$ treatment groups (during 1st TRAINING - before treatment), learned the place avoidance in the AAPAT worse than young non-treated rats assigned to the respective vehicle or $\mathrm{D}+\mathrm{Q}$ treatment groups and exhibited short-term memory impairment. However, all rats improved place avoidance across training days. The two-way ANOVA (groups $n=4$ vs. days $n=5$ ) for number of entrances and shocks confirmed effect of training days (entrances $\mathrm{F}_{(4,104)}=12.09 ; p<0.001$; shocks $\mathrm{F}_{(4,100)}=23.19 ; p<0.001$ ) (Figure 1B I, 1C I). The number of entrances and shocks decreased after D2 (Tukey's multiple comparisons test; $p<0.001$ ). The post hoc Tukey's test for interaction groups vs. days $\left(\mathrm{F}_{(12,100)}=3.23 ; p<0.001\right)$ for shocks confirmed that during the first training day all aged rats received more shocks than all young rats $(p<0.001)$, whereas on the last training day this difference was not significant (Figure 1B I, 1C I, 1E I).

Aged rats improved place avoidance acquisition and short-term memory after administration of D+Q.

\section{Supplementary Data 2A}

After 8 weeks of $D+Q$ or vehicle administration the 2 nd TRAINING of place avoidance was repeated with the new location of the shock place (2nd TRAINING) (Figure $1 \mathrm{~A}$ ). $\mathrm{D}+\mathrm{Q}$ treatment improved cognitive ability only in aged rats. However, two way ANOVA (groups $n=2$ vs. days $n=5$; aged VEH 2 nd TRAINING, aged $\mathrm{D}+\mathrm{Q}$ 2nd TRAINING vs. 5 days) confirmed significant day effects for entrances $\left(\mathrm{F}_{(4,40)}=5.07 ; p=0.002\right)$, shocks $\left(\mathrm{F}_{(4,44)}=4.39 ; p=0.004\right)$, maximum time avoided $\left(F_{(4,43)}=4,106\right)$. Rats made more entrances on D1 than on D3, D4 and D5, received more shocks and presented shortened maximum time avoided on D1 than on D3 and D4 (Tukey's multiple comparisons test; $p<$ 0.01) (Figure 1B II, 1C II, 1E II).

\section{Supplementary Data 2B}

Old rats from the $\mathrm{D}+\mathrm{Q}$ group during 2nd TRAINING compared to their own results from the 1st TRAINING showed significant day effect for entrances (ENTR $\left.\mathrm{F}_{(4,43)}=6.37 ; p<0.01\right)$, shocks $\left(\mathrm{F}_{(4,43)}=5.86\right.$; $p<0.001)$, shocks/entrances ratio $\left(\mathrm{F}_{(4,43)}=1,09\right.$; $p<0.01)$ and maximum time avoided $\left(\mathrm{F}_{(4,44)}=3.73 ; p\right.$ $=0.01)$. Rats made more entrances and received more shocks on D1 than in the next training days (Tukey's multiple comparisons test; $p<0.01)$. Skill ratio was on a lower level on D5 than on D1 $(p<0.009)$.

\section{Supplementary Data 3}

$\mathrm{D}+\mathrm{Q}$ treatment did not influence performance of young rats neither when compared to the young vehicle group nor to their own results from the 1st TRAINING. Although effect of groups was not significant but the two way ANOVA (groups $n=2$ vs. days $n=5$ ) showed a significant effect of days for entrances $\left(\mathrm{F}_{(4,56)}=3.52\right.$; $p<0.012)$, shocks $\left(\mathrm{F}_{(4,56)}=6.34 ; p<0,001\right)$, SHs/ENTRs ratio $\left(\mathrm{F}_{(4,56)}=4.82 ; p=0.002\right)$. The number of entrances on D4 and on D5 was lower than on D1 (Tukey's multiple comparisons test; $p<0.02$ ), the number of shocks decreased after D1 $(p<0.001)$ and SHs/ENTRs ratio was lower on D4, D5 than on D1 $(p=0.003)$ (Figure 1B II, 1C II, 1D II).

\section{Supplementary Data 4}

Young rats before and after $\mathrm{D}+\mathrm{Q}$ administration changed performance in consecutive days of training. The two way ANOVA (1st TRAINING, 2nd TRAINING vs. days $n=5$ ) confirmed day effect for entrances $\mathrm{F}_{(4,56)}=3.37 ; p=0.015$; shocks $\mathrm{F}_{(4,56)}=6.22$; $p<0,001$; SHs/ENTRs $\left.\mathrm{F}_{(4,56)}=3.92 ; p=0.007\right)$. On D1 rats made more entrances, received more shocks and presented a high ratio than in the next training days (Tukey's multiple comparisons test; $p<0.01$ ) (Figure 1B I, 1C I, 1D I).

$\mathrm{D}+\mathrm{Q}$ alleviates age-associated cognitive deficits in aged rats at least 5 weeks after discontinuation of the treatment.

\section{Supplementary Data 5}

Similarly to the first set of experiments we found that aged rats learned the place avoidance task worse than young rats before treatment with $\mathrm{D}+\mathrm{Q}$ during the $1 \mathrm{st}$ TRAINING. However, the two-way ANOVA (2 groups and 5 days) for entrances, shocks and maximum time avoided on the 1st TRAINING confirmed effect of days (entrances $\mathrm{F}_{(4,44)}=41.70 ; p<0.0001$; shocks $\mathrm{F}_{(4,48)}=$ 49, $29 P<0,0001$; maximum time avoided $\mathrm{F}_{(4,48)}=$ $9,293 p<0,0001$ ) (Figure 5B I, 5C I, 5D I).

All rats improved performance across days and presented less entrances, shocks and longer maximum 
time avoided on D3, D4 and on D5 compared to D1 and D2 (Tukey's test $p<0.01$ ). Improvement of performance over the course of days is evidence that tested rats retained the ability to learn and memorize despite their age. It was confirmed by non-significant group effect for skill learning evaluated as SHs/ENTRs ratio, whereas the day's effect was significant (two-way ANOVA $\left.\mathrm{F}_{(4,48)}=29.60 ; p<0.001\right)$. Rats improved skills from the third day of 1 st TRAINING. Moreover, there was a significant groups vs. days interaction for entrances $\left(\mathrm{F}_{(4,44)}=3.95 ; p=0.008\right)$ and shocks $\left(\mathrm{F}_{(4,48)}=\right.$ 4.58; $p=0.003)$. Aged rats on D2 and D3 made more entrances than young rats, whereas on D1 and D2 received more shocks than young rats at the same time points (the Tukey's multiple comparisons test; $p=0.01$ ) (Figure 5B I, 5C I, 5D I).

\section{Supplementary Data 6}

The 2nd TRAINING of AAPAT with the new location of the to-be-avoided place started after 8 weeks of $\mathrm{D}+\mathrm{Q}$ administration. During this training aged rats exhibited memory improvements relative to their own results from the 1st TRAINING (Figure 5B I, 5C I, 5D I, 5E I). Moreover the two-way ANOVA (2 trainings 1st TRAINING, 2nd TRAINING and 5 days) for the number of entrances, shocks, skill learning and maximum time avoided confirmed effect of days (entrances $\mathrm{F}_{(4,48)}=40,06 ; p<0,001$; shocks $\mathrm{F}_{(4,48)}=38.14 ; p<0.001 ;$ SHs/ENTRs $\mathrm{F}_{(4,48)}=19.09$; $p<0.001 ;$ maximum time avoided $\mathrm{F}_{(4,48)}=13.37 ; p<$ $0.001)$. The animals made fewer entrances with longer maximum time avoided on D4, D5 and received a lower number of shocks on D4 and on D5 than on D1, D2 and D3 (Tukey's multiple comparisons test; $p<0.001$ ). Old $\mathrm{D}+\mathrm{Q}$ treated rats presented improvement in skill learning on D1 and on D5 of 2nd TRAINING in comparison to the 1st TRAINING (post-hoc test $p<0.001$ and $p<0.001$ for SHs/ENTRs for interaction training vs. days $\mathrm{F}_{(4 \text {, }}$ 48) $=7.517 ; p<0.001)$. Rats entered the to-beavoided place more often during the 1st TRAINING than during the 2nd TRAINING (post hoc $p<0.001$ for interaction trainings vs. days $\left(\mathrm{F}_{(4,48)}=9,80 ; p<\right.$ $0,001)$. Numbers of shocks on D1 and on D2 were higher for 1st TRAINING than for the 2nd TRAINING (post hoc $p<0.001$ for interaction trainings vs. days $\left.\mathrm{F}_{(4,48)}=25,00 ; p<0,001\right)$.

\section{Supplementary Data 7}

The young rats have shown a lack of significant differences between 1st TRAINING and 2nd TRAINING for entrance, shocks, skill learning and maximum time avoided (Figure 5B I, 5C I, 5D I, 5E I). However, similarly to the aged rats, the young rats improved performance in AAPAT across days of training (entrances $\mathrm{F}_{(4,48)}=20.41 ; p<0.001$; shocks $\mathrm{F}_{(4,}$ ${ }_{48)}=20.38 ; p<0.001 ; \mathrm{SHs} / \mathrm{ENTRs} \mathrm{F}_{(4,48)}=6.09 ; p=$ 0.002 ; maximum time avoided $\mathrm{F}_{(4,48)}=4.99 ; p=0.002$ ). The number of entrances decreased significantly from D2 to D5 in comparison to D1 (Tukey's multiple comparisons test; $p<0.001$ ), whereas SHs/ENTRs ratio was low on D5 in comparison to D1 $(p=0.006)$. Tmax was long in the last three days of training.

Interaction training vs. days was significant for the number of shocks $\left(\mathrm{F}_{(4,48)}=4,96 ; p=0.002\right)$. The post hoc test confirmed that the young rats on D1 of the 1 st TRAINING received a greater number of shocks than on D1 in the 2nd TRAINING furthermore, the number of shocks was decreasing in consecutive days of each training compared to D1 $(p<0.001)$.

\section{Supplementary Data 8}

Results of the 3rd TRAINING revealed that aged rats retained improvements in cognitive abilities at nearly the same level as immediately after $D+Q$ treatment (2nd TRAINING). No significant differences were found between 2nd and 3rd TRAINING (Figure 5B III-5E III). However, a significant decrease in the number of entrance, the number of shocks, a low level of SHs/ENTRs ratio and an increase of maximum time avoided were recorded during 3rd TRAINING in comparison to the 1st TRAINING. The two way ANOVA ( 2 trainings 1 st TRAINING, 3rd TRAINING and 5 days) confirmed significant effect of days (entrances $\mathrm{F}_{(4,48)}=27.68 ; p<0.001$; shocks $\mathrm{F}_{(4,48)}=$ $42.50 ; p<0.001 ; \mathrm{SHs} /$ ENTRs $\left(\mathrm{F}_{(4,48)}=12.47 ; p<\right.$ 0.001 ; maximum time avoided $\mathrm{F}_{(4,48)}=8.14 ; p<$ $0.001)$. Rats made less entrances, received less shocks and improved skills on D3, D4 and D5, compared to D1 and D2, whereas maximum time avoided was longer on D5 than on D1 and D2 (Tukey's multiple comparisons test; entrances $p=0.01$; shocks $p=0.01$; skills $p<0.001$; maximum time avoided $p=0.01$ ). Old rats on $\mathrm{D} 1$ and on $\mathrm{D} 2$ receive more shocks than during the next days of the 1st TRAINING and all days of the 3rd TRAINING (post hoc $p<0.001$; for interaction training vs. days $\left.\mathrm{F}_{(4,48)}=18.84 ; p<0.001\right)$.

\section{Supplementary Data 9}

Due to the long duration of the experiments, a group of young rats reached approximately 7-8 months of age at the end of 3rd TRAINING when the final memory training to a new to-be-avoided place was performed. No differences in place avoidance was found in the group of young $\mathrm{D}+\mathrm{Q}$ treated rats on 3rd TRAINING in comparison to 2nd TRAINING. However, the two way ANOVA (2 trainings 1st TRAINING, 3rd TRAINING 
and 5 days) for trainings confirmed significant effect of days for entrances $\left(\mathrm{F}_{(4,44)}=23.70 ; p<0.001\right)$, shocks $\left(\mathrm{F}_{(4,44)}=21.85 ; p<0.001\right) \mathrm{SHs} /$ ENTRs $_{(3,206)}=6.288$; $p=0.001$; maximum time avoided $\mathrm{F}_{(4,44)}=5.46 ; p=$ 0.001). (Figure 5B II-5E II). On D1 rats made more entrances, received more shocks and presented higher SHs/ENTRs ratio than in the next few days (Tukey's multiple comparisons test; $p<0,001$ ), whereas maximum time avoided significantly increased after D1 $(p<0.001)$. 
A

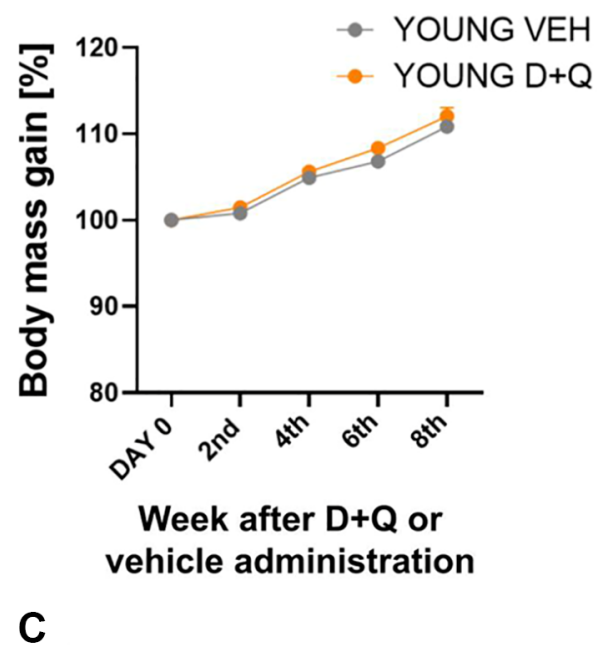

C

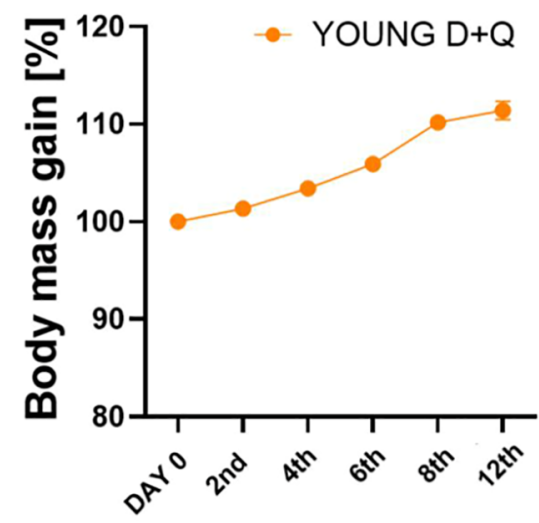

Week after $D+Q$ or E vehicle administration

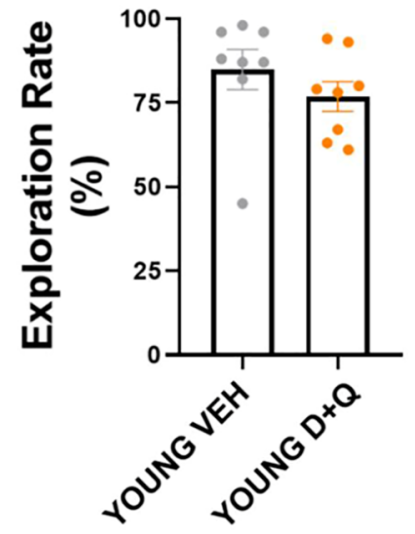

B

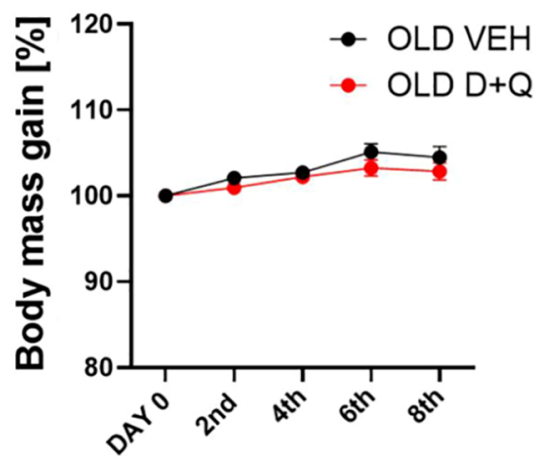

Week after $D+Q$ or vehicle administration

D

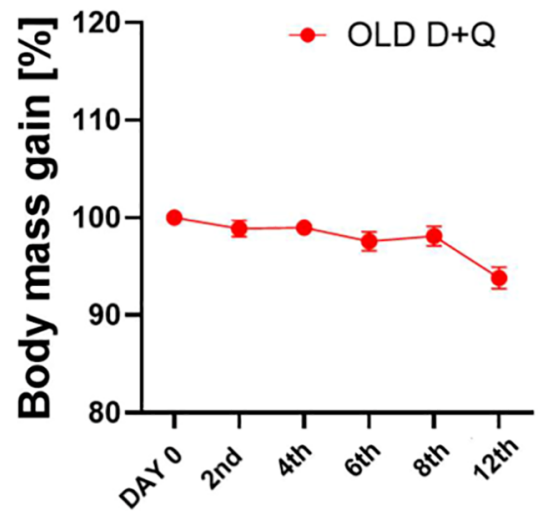

Week after $D+Q$ or

$\mathbf{F}$ vehicle administration

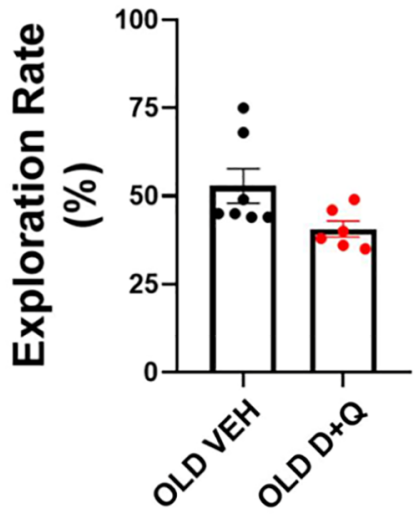

Supplementary Figure 1. D+Q treatment does not change long-term memory retrieval, locomotor activity in male Wistar rats. Relative body mass gain throughout the experiments was measured in relation to the body mass recorded immediately before administration of the first dose of $D+Q$ or vehicle (VEH) (A-D). Exploration rate in open field test was performed after the $D+Q$ or vehicle treatment in young $(E)$ and aged $(\mathbf{F})$ animals. Exploration ratio was calculated by dividing the open field arena into $10 \mathrm{~cm}^{2}$ squares and calculating the ratio of explored squares recorded with the Toxtrac software over the total number of squares for every individual of both mischiefs during the open field test. Data were analyzed by Two-way ANOVA followed by Tukey multiple comparison test for vehicle vs. $D+Q$ groups before and after treatment with ${ }^{*} p<0.05$. The results are expressed as mean $\pm \operatorname{SEM}, n N=7-8$. 

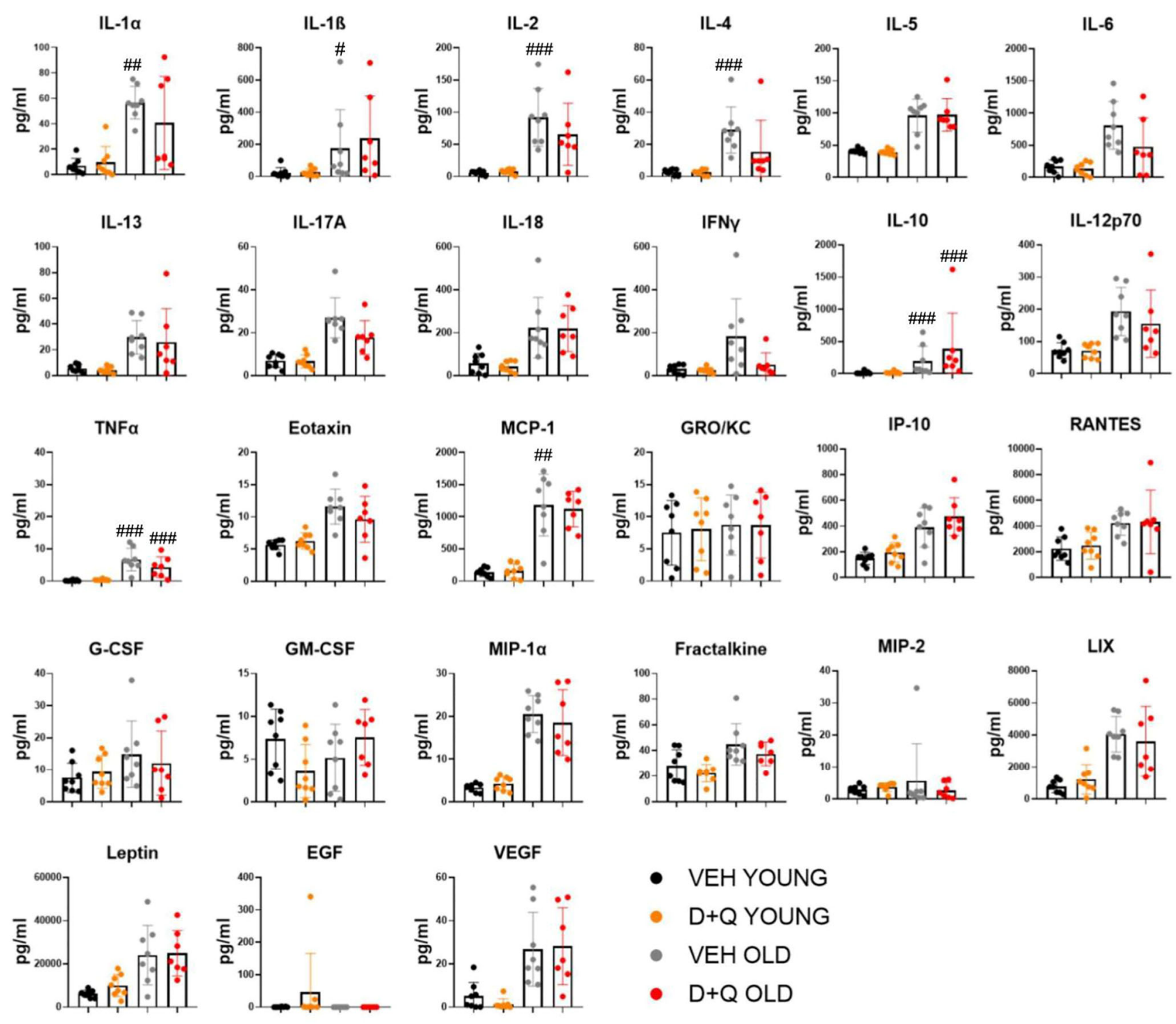

Supplementary Figure 2. Levels of each of the analytes measured in blood serum collected from young and aged animals after 8 week of $D+Q$ or vehicle (VEH) administration. Each dot represents a mean value from two technical replicates. The results are

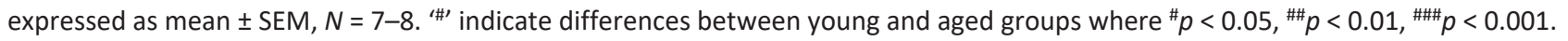


A

OLD VEH

OLD $D+Q$

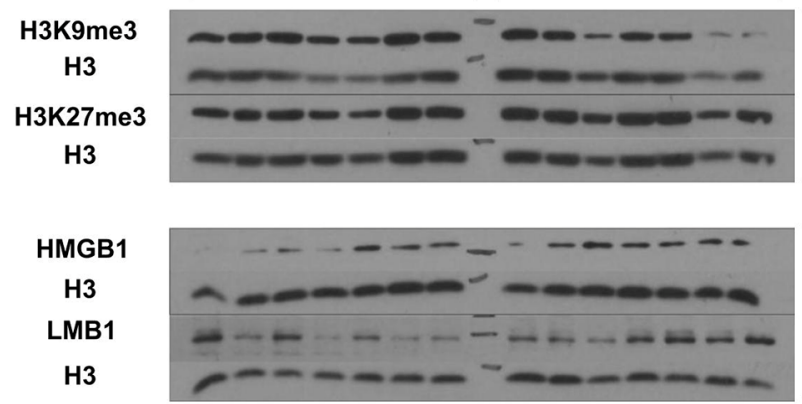

B

Hippocampus (nuc. fraction) LMB1/H3

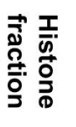

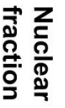

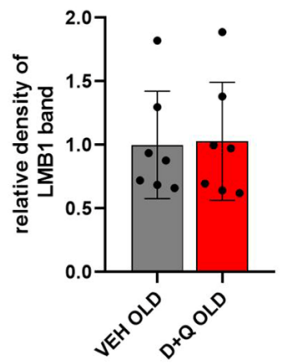

C Hippocampus (nuc. fraction) HMGB $1 / \mathrm{H} 3$

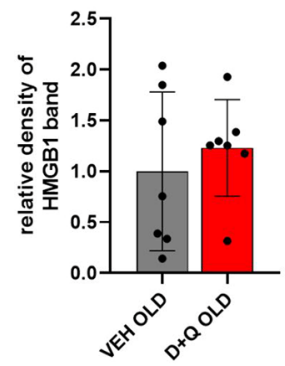

Supplementary Figure 3. Western blot results (A) Raw scans of Western blots of HMGB1, LMB1, H3K9me3 and H3K27me3 together with corresponding histone H3 level. Relative density of LMB1 (B) and HMGB1 (C) measured in the nuclear fraction of hippocampal homogenate from young and aged animals after 8 week of $D+Q$ or vehicle (VEH) administration. Each dot represents the value obtained from one animal. The results are expressed as mean \pm SEM. Results were normalized to the mean of the aged vehicle group $(O L D V E H), N=7$.

A
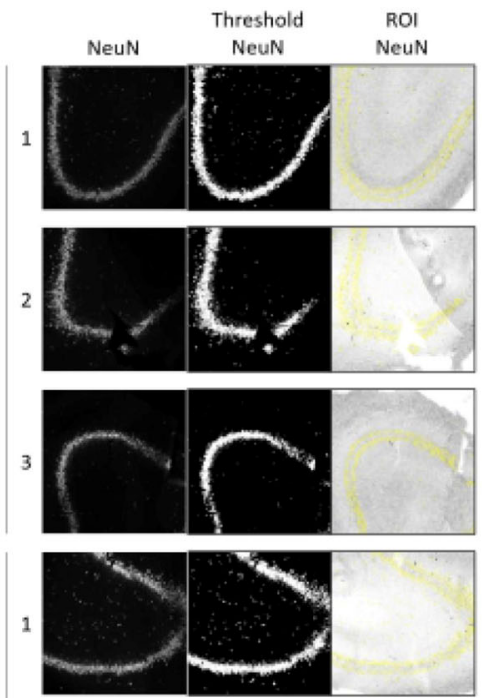

$D+Q$
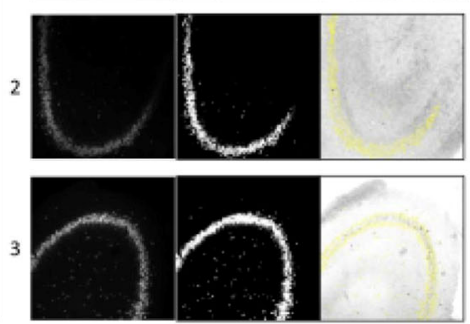
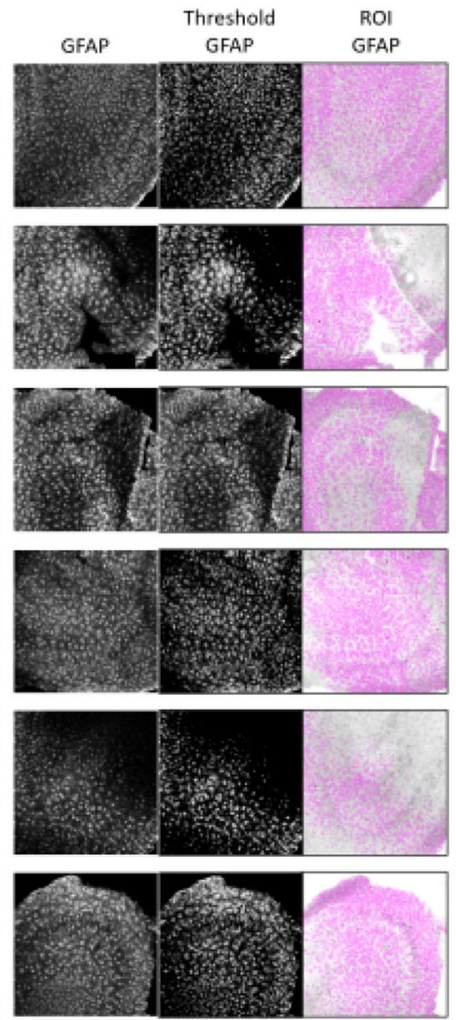
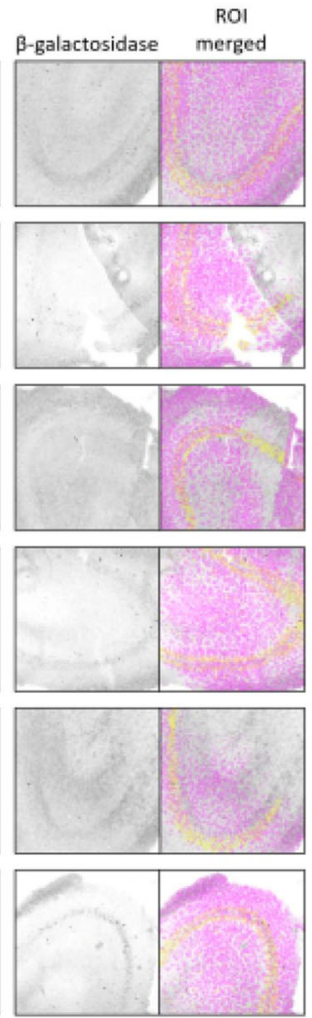
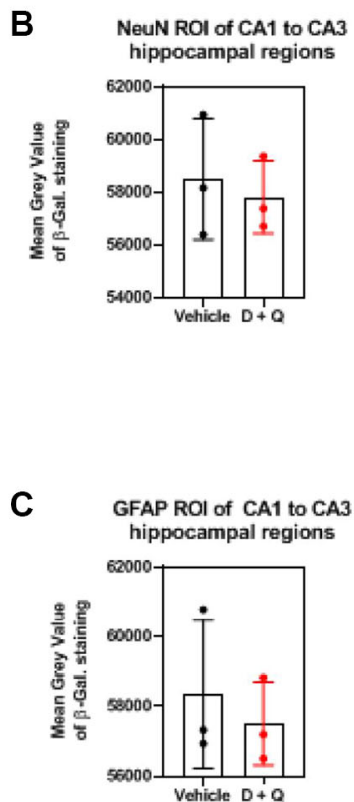

Supplementary Figure 4. SA- $\beta$-galactosidase expression in hippocampal CA1 to CA3 region of vehicle and D+Q treated rats. PFA fixed brain of vehicle and $D+Q$ treated rats were cut in $30 \mu \mathrm{m}$ slices. Hippocampi were isolated and $\mathrm{SA}-\beta$-galactosidase staining was performed. Astrocytes and neurons were targeted by immunofluorescence prior to SA- $\beta$-galactosidase staining with either anti-GFAP or anti-NeuN antibodies to define regions of interest by thresholding the signal intensity using Fiji (ImageJ) software. Within each region of interest, mean grey value was measured using the same software. Representative images for each rat from both treatment groups showing sum projection of NeuN, GFAP and REST staining, thresholding of NeuN and GFAP along with the overlay of their individual and merged regions of interest (A). Mean grey value of SA- $\beta$-galactosidase staining in the NeuN region of interest from CA1 to CA3 hippocampal region (B). Mean grey value of SA- $\beta$-galactosidase staining in GFAP region of interest from CA1 to CA3 hippocampal region (C). Data were expressed as scattered dots bar-plots showing mean \pm SEM. $N=3$ rats per group comprising 2 images of individual hippocampal slices per animal across two separate immunofluorescence experiments. Data were determined by Student $t$-test between vehicle and $D+Q$. 